\title{
Combining Reflexes and External Sensory Information in a Neuromusculoskeletal Model to Control a Quadruped Robot
}

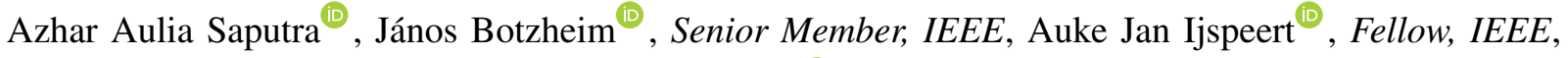 \\ and Naoyuki Kubota ${ }^{\mathbb{D}}$, Member, IEEE
}

\begin{abstract}
This article examines the importance of integrating locomotion and cognitive information for achieving dynamic locomotion from a viewpoint combining biology and ecological psychology. We present a mammalian neuromusculoskeletal model from external sensory information processing to muscle activation, which includes: 1) a visual-attention control mechanism for controlling attention to external inputs; 2) object recognition representing the primary motor cortex; 3) a motor control model that determines motor commands traveling down the corticospinal and reticulospinal tracts; 4) a central pattern generation model representing pattern generation in the spinal cord; and 5) a muscle reflex model representing the muscle model and its reflex mechanism. The proposed model is able to generate the locomotion of a quadruped robot in flat and natural terrain. The experiment also shows the importance of a postural reflex mechanism when experiencing a sudden obstacle. We show the reflex mechanism when a sudden obstacle is separately detected from both external (retina) and internal (touching afferent) sensory information. We present the biological rationale for supporting the proposed model. Finally, we discuss future contributions, trends, and the importance of the proposed research.
\end{abstract}

Index Terms-External sensory information, neurocognitive locomotion, neuromusculoskeletal model.

\section{INTRODUCTION}

$\mathbf{H}$ UMANS and other animals have dynamic movement abilities adapted to complex environments. They move efficiently by integrating many aspects of their morphology and central nervous system. External (visual) and internal

Manuscript received April 22, 2020; revised September 13, 2020; accepted January 2, 2021. This work was supported in part by the Grant-in-Aid for Scientific Research from Japan Society for the Promotion of Science under Grant 18J21284. The work of János Botzheim was supported by the János Bolyai Research Scholarship of the Hungarian Academy of Sciences. This article was recommended by Associate Editor X. Zhu. (Corresponding author: Azhar Aulia Saputra.)

Azhar Aulia Saputra and Naoyuki Kubota are with the Graduate School of System Design, Tokyo Metropolitan University, Tokyo 191-0065, Japan (e-mail: azhar-aulia-saputra@ed.tmu.ac.jp; kubota@tmu.ac.jp).

János Botzheim is with the Department of mechatronics, Optics, and Mechanical Engineering Informatics, Budapest University of Technology and Economics, 1111 Budapest, Hungary (e-mail: dr.janos.botzheim@ @ieee.org).

Auke Jan Ijspeert is with the Biorobotics Laboratory, Ecole Polytechnique Féderalé de Lausanne, 1015 Lausanne, Switzerland (e-mail: auke.ijspeert@epfl.ch).

Color versions of one or more figures in this article are available at https://doi.org/10.1109/TCYB.2021.3052253.

Digital Object Identifier 10.1109/TCYB.2021.3052253 (somatosensory) sensory information inform an animal's decision to move. Likewise, a legged robot also needs a dynamic locomotion generator if it is to cope with diverse environmental conditions. The need for such a locomotion model connects the ideas of biologists with those of roboticists. Robot developers try to mimic human or animal locomotion through the locomotion generator's design. Biology-inspired models for generating locomotion include those with central pattern generation (CPG) [1]-[3], and may include muscle models as the target actuator [4]. However, current models still have not achieved dynamic locomotion faithful to that of humans or other animals. A useful review of the current issues and challenges in bioinspired-based locomotion is provided in [5]-[7].

Locomotion is complicated. In addition to the actual motion generated, locomotion integrates all balancing, reasoning, perception, memorization, and embodiment. Locomotion behavior can be affected by both external conditions and internal sensory information. For example, when a legged animal moves directly forward and encounters an obstacle, the animal will change its movements to avoid that obstacle. Specifically, when we swing our legs while walking, we might suddenly notice an obstacle where we were about to step. At that moment, we immediately try to change our leg's swing to land on a safe area. These illustrations demonstrate a role for cognition in the locomotion model. The need for cognition is supported from psychological and neurophysiological viewpoints [8]-[10].

Legged locomotion research draws together numerous underlying principles, but it is still unclear how these principles are integrated into animal motor control. It is therefore essential to analyze their natural integration. Animals have a complex neuron structure for generating locomotion. The basic locomotion pattern is generated by CPG in the spinal cord. From there, the pattern stimulates other neural pools toward muscle synergies [11]-[13]. This spinal reflex process contributes the overall movement pattern, muscle activation, and the modulation of CPG. In quadrupeds, CPG generates different gait patterns in walking, trotting, pacing, and galloping [14]. Spinal reflexes are able to integrate the mechanical sensory information with the muscle activation via alpha motor neurons without involving central inputs. There are also other reflexes, such as the postural reflex, that affect motion generation. The postural reflex maintains the body's posture 
and stability. The postural reflex manages the information of somatosensory stimuli from the body [15]. It is similar to the stumbling reflex: when our foot falls on an unstable stone while walking, the leg is automatically lifted in response. It then swings rapidly forward and extends, ready to provide support as we fall. The postural reflex similarly allows us to avoid suddenly encountered obstacles before making contact. However, the postural reflex is more complicated; it involves the cognitive system. Therefore, the satisfactory generation of dynamic locomotion must consider the role of cognitive information.

Building on current thinking about the integration of cognitive information and muscle reflex, we address three questions: 1) how does the reflex system respond to cognitive information when perceiving a sudden obstacle? 2) what role does visual attention play in generating locomotion? and 3) how can a biological model be implemented in a legged robot? Our overall goal is to realize benefits from biological modeling in developing a locomotion model for a legged robot. Specifically, this article develops a novel locomotion model by integrating the supraspinal level from cognitive information and muscle reflex system to achieve dynamic locomotion controller for a quadruped robot. We design this model by mimicking the descending flow of information from vision to muscle activation, and focus especially on a control mechanism for short-term adaptation when suddenly perceiving an obstacle.

This article is organized as follows. Section II shows the general concept of the proposed model. Then, Section III details the cognitive model from its external sensory input model until it outputs information to $\mathrm{CPG}$, via a particular attention control model, affordance detection, and a commanding model. In Section IV, we proposed a multilayer CPG model. After that, Section V explains the felidae-like hindlimb and forelimb muscle model used. In order to prove the reliability of the proposed model, we show several appropriate experiments in Section VI. In Section VII, we show the result of the proposed model using a quadruped robot. Finally, we conclude and discuss further development in Section VIII.

\section{A. Information From the Supraspinal Level to Motor Neurons}

In order to analyze the involvement of cognitive processing at the supraspinal level, researchers have studied corticospinal tract activity. When there is no obstacle, corticospinal activity is low. When an obstacle appears during walking, corticospinal activity rises during the leg-swing phase [16]. The animal has to visually regulate its limb trajectory to avoid the obstacle and to position the limb in a safe location [8]. At the limb, there is also a significant increase in motor cortex activity during high-attention movement, such as in narrow passages, over rough terrain, when avoiding obstacles, and especially during swing phases that require precise foot placement by controlling limb trajectory [17]-[23]. Those observations demonstrate the effect of visual information on locomotion through motor cortical discharge. Further insight is given by red nucleus neuron activity in walking cats. Activity increases when cats step over obstacles [24]. This increase shows that the red nucleus also affects how muscle activity patterns are modified. The red nucleus signals via the rubrospinal tract to primarily affect the forelimb or upper limb, and performs a lesser role than the corticospinal tract [25], [26]. However, the transfer of visual information from the occipitoparietal area into the corticospinal tract is not clearly understood [27].

\section{B. Motor Neuron Command by Cognitive Information During Obstacle Avoidance}

Neuro physiological studies have found several strategies for facing obstacles, for different environmental conditions. Chu et al. [28] analyzed the behavior of cats avoiding obstacle during walking. They found that cats tend to change their muscle activity without altering the overall locomotion pattern. This shows the effectiveness of the neuromotor process. Humans also show similar behavior when facing obstacles, tending to maintain the phase duration or locomotion pattern, preferring instead to alter muscle activity [29]. This strategy is supported by the CPG model proposed by Rybak et al. that commands the motoneuronal pools for directly controlling muscle, bypassing the rhythm generator in the spinal neural network [13], [30], [31]. In contrast, when forced to step over an obstacle, both humans and cats tend to control the trajectory of swing as well as its duration. In cat locomotion, stimulation from both the red nucleus and the motor cortex affect flexor muscles in the swing phase [25], [32].

\section{SySTEM DESIGN}

The aim of our proposed model is to build a dynamic locomotion model in quadruped robots that achieves better locomotion control than alternative approaches. We imitate animal locomotion processes from neurophysiology and ecological psychology. We incorporate influences from the cognitive process in changing locomotion behavior, such as when avoiding an obstacle, and especially when avoiding a sudden obstacle. In the human neural control-based locomotion process, a locomotion command specifying a placing reflex or a movement provision command descends from higher centers (the brain) to the mesencephalic locomotor region (MLR), which drives the CPG system for controlling muscle activation.

Fig. 1 outlines how we mimic that top-down mechanism. The mechanism begins with the processing of external sensory information. Attention is controlled by the superior colliculus (SC) with command from the cortex. The cortex builds an understanding of the environment using external sensory information from the retina. The movement-related command will then be passed to the MLR. Information from the MLR transfers to the CPG through the corticospinal tract and reticulospinal tract. The corticospinal tract tends to control fine movement, such as a specific position, while the reticulospinal tract is mainly involved in gross control, such as the locomotion pattern. The mimetic process of the above mechanism will be detailed in Section III.

Next, based on the information transmitted through the corticospinal tract, the CPG generates the appropriate cadence and 


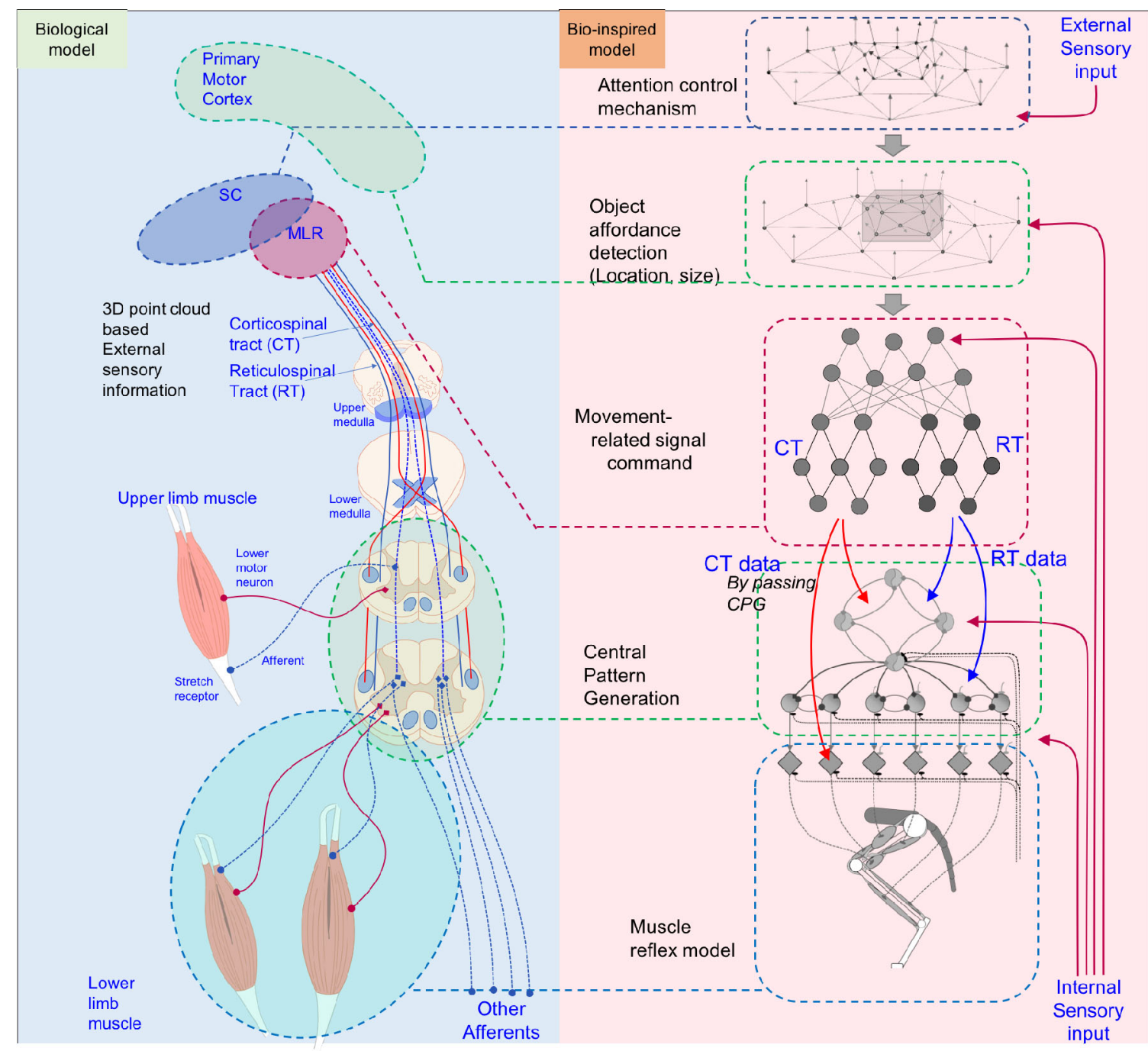

Fig. 1. Design of the motion control pathway from neurophysiological and ecological psychological viewpoint.

phase durations, and the pattern formation $(\mathrm{PF})$ layer modulates the motoneuronal outputs. We elaborate the single rhythm generator model previously proposed by Rybak et al. [13] A single-rhythm generator network controls the activity of multiple-unit PF modules connected to subpopulations of motoneurons. The spinal motor neural pools then process this output into a spike-based signal. This spike-based signal regulates the strength of the muscle activation phase; details will be given in Section IV. Furthermore, we also build the muscle reflex model for the forelimb and hindlimb, which will be described in Section V. Internal sensory information will affect the movement command, CPG, and muscle reflex system.

\section{Cognitive Process to Movement-Related TRACT}

In this section, we propose a process from cognition to the movement-related tract, based on a top-down model in mammals. Cognition involves the perception of external sensory information. It also includes a way to control the attention to external information for movement behavior. External sensory information plays a big role in controlling locomotion behavior. Visual information is received and processed by the retina and optic nerve and transferred to the thalamus and SC. The SC contributes to coordinating eye movement, responding to strong gaze direction commands from the cortex. The thalamus passes visual information to the cortex. Most studies show that there are two processing streams in the cortex: 1) the ventral system (the "what" pathway) for recognizing and identifying the object, and 2) the dorsal system (the "where" pathway) for identifying the object's location and motion. The dorsal system is also involved in visually guided locomotion [33]. Movement-related information from the motor cortex is carried to the spinal cord by the corticospinal tract.

In order to represent this abstraction mechanism, we draw on neurophysiology. First, we build a dynamic attention model explained in Section III-A to represent the gaze. To recognize objects in front of the robot, we build an object affordance detector as explained in Section III-B. This detector provides the object size, location, and movement, providing for possible action at lower levels. Furthermore, we develop an interaction between the SC and the motor cortex. Our model connects the dynamic attention model with the affordance detection model for controlling the gaze or attention area. 


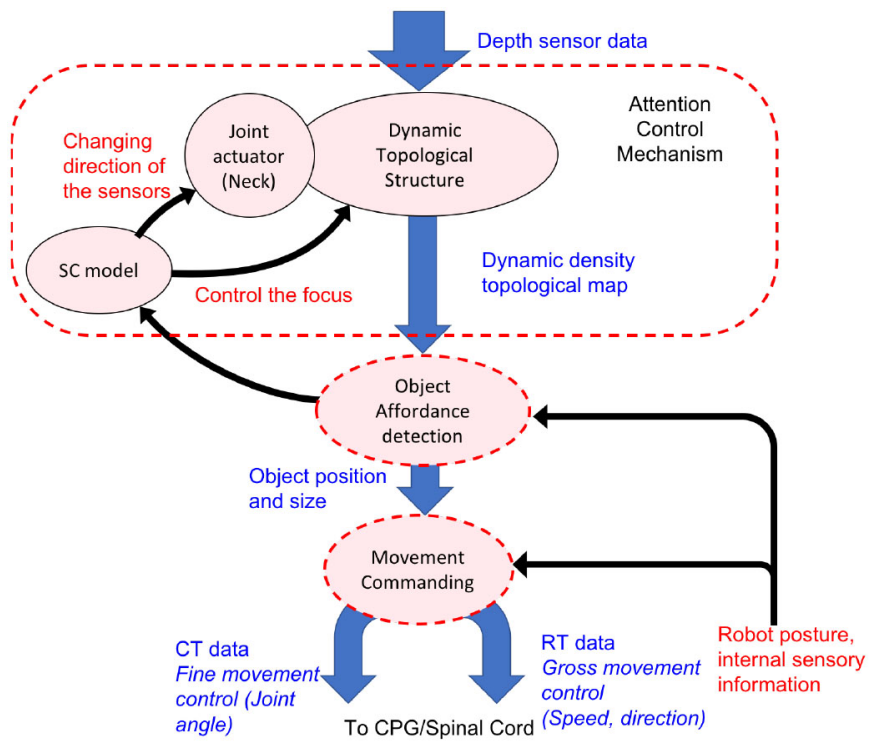

Fig. 2. Design of the attention control mechanism and process of the external sensory information pathway until becoming movement-related information.

\section{A. Dynamic Attention}

During movement, gaze attention is dynamic and strongly integrated with the surrounding environment. To model that, we imitate the SC mechanism. The SC receives data from ganglion cells in the retina. We use time-of-flight sensors to provide external sensory information, as in our previous work [34], [35]. The aim of our model is to combine the attention and action which is effectively implemented for sudden obstacle response. Visual information requires a large amount of processing and knowledge building to estimate the shape and size of the object. On the other hand, the 3 -D point cloud information can provide the vector position directly which makes easier to recognize the shape of some object. Therefore, point cloud information can be used directly without much processing, for example, without object recognition. Topological map structure comprising neurons connected with their neighbors can also reduce the memory usage. The topological map represents the attention, with neuron density in this map representing the attention level. Fig. 2 shows how the SC model controls the coordination between neck joint and the focus or density of the topological structure entering the object affordance detection system. We consider the neck joint in roll direction $\left(\theta_{\text {neck }}\right)$ by calculating $\dot{\theta}_{\text {neck }}=\tanh \left(\left(\theta_{\text {off }}-\theta_{\text {neck }}\right)+\left(M_{\text {off }}-M_{y}\right)^{3}\right) \cdot \exp \left(\ln (0.5) \cdot \theta_{\text {neck }}^{2}\right)$, where $\theta_{\mathrm{off}}, M_{\mathrm{off}}$, and $M_{y}$ are the default value of neck joint angle, the center of frame in y-direction, and the attention position of the frame in $y$-direction, respectively. The SC model will select the area where an obstacle blocks the movement. It controls the attention by adjusting the number of neurons in associated areas and coordinating the neck joint to avoid the attention in the edge area of the gaze. The illustration of the attention model is shown in Fig. 3. In flat terrain, the normal vector $(\mathbf{N})$ is homogeneous. The model has low attention where the nodes are sparse. When we put a sudden red box, then few nodes are shifted. There are nonhomogeneous

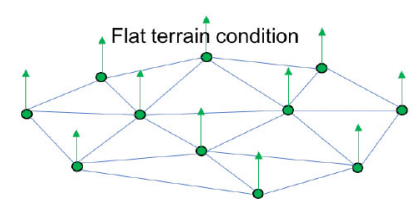

Normal vector of nodes are uniform Robot perceives safe condition
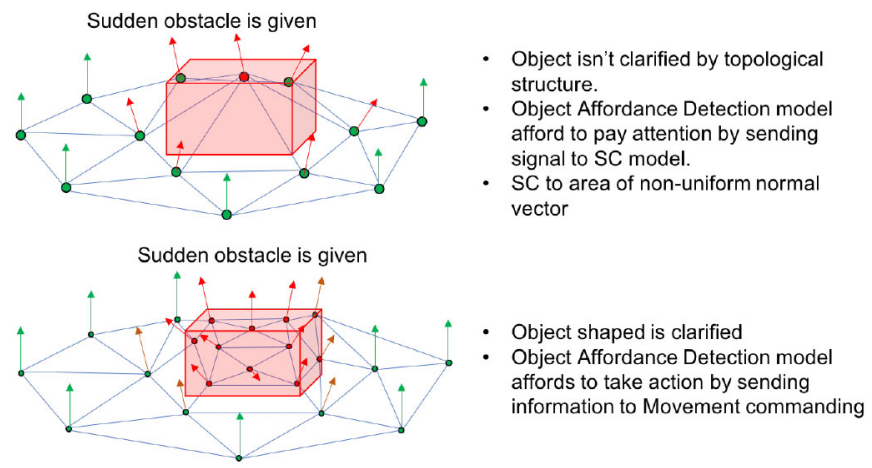

Fig. 3. Attention system using a topological map model.

normal vectors (red color). In this state, the object still cannot be detected precisely. Then, the attention increases the number of nodes around the nonhomogeneous node. After the nodes are increased, then the object is clarified. The information is enough for further processes.

Our topological structure is built on a dynamic density growing neural gas model (DD-GNG) [36]. Local node density is set by controlling the probability of finding raw data in that neighborhood. We hence calculate the strength of each node as influenced by the attended area. Node strength is calculated using (1) for the discount rate, while (2) controls the probability of finding information by local random search. Parameter $S(P)$ represents the value of random search probability of a set of raw data $(P)$. The parameter $\mathbf{h}$ is all nodes's position and $h_{k}$ represents the $k$ th 3 -D node's position; $r$ and $\mu$ are the radius of the sphere, and the 3-D position of the sphere received from the affordance detection

$$
\begin{aligned}
\delta_{k} & =\frac{e^{-\left(r+\left\|h_{k}-\mu\right\|\right)}}{1+e^{-\left(r+\left\|h_{k}-\mu\right\|\right)}} \\
S(P) & =0.2+\frac{e^{-(r+\|P-\mu\|)}}{1+e^{-(r+\|P-\mu\|)}} .
\end{aligned}
$$

The attended area will be selected and calculated by the affordance detection model that represents the cortex's main role. The main algorithm is shown in Algorithm 1.

\section{B. Object Affordance Detection}

The concept of affordance was proposed by Gibson [37] in ecological psychology. Affordances connect between environment and individual, and also represent possible actions depending on the individual's capabilities [38]. In this model, affordance detection will provide possible actions. There are two feedback flows involving object affordance detection in the cortex. The first one commands the SC to control the appropriate attention. The second one commands the spinal cord and corticospinal tract.

In our model, we calculate the affordance from the vectors of available edges in every node $(N)$ calculated in the following 


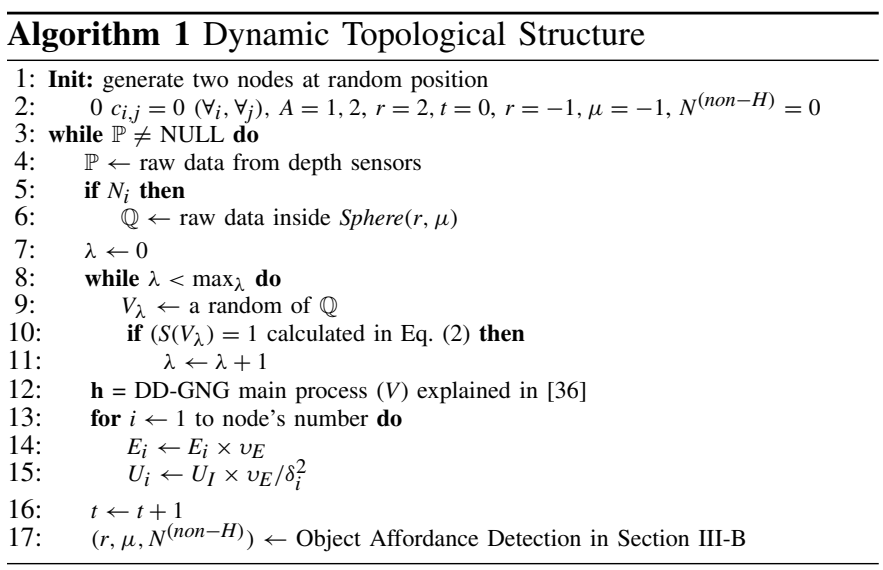

equation:

$$
\mathbf{N}=\frac{1}{N_{i}^{(\text {edge })}} \sum_{k=0}^{k=N_{i}}\left(\frac{\left(\mathbf{h}^{i}-\mathbf{n}_{k}^{i}\right) \times\left(\mathbf{h}^{i}-\mathbf{n}_{k+1}^{i}\right)}{\|\left(\mathbf{h}^{i}-\mathbf{n}_{k}^{i}\right) \times\left(\mathbf{h}^{i}-\mathbf{n}_{k+1}^{i} \|\right.}\right)
$$

where $N_{i}$ and $\mathbf{h}^{i}$ are the number of edges and vector node position in the $i$ th node. $\mathbf{n}_{k}^{i}$ is the vector position of the node connected with the $k$ th edge in the $i$ th neuron.

1) Representing Cortex and SC Integration: When the density of node $(\alpha)$ in the nonhomogeneous vector $\left(N^{(\text {non-H) }}\right)$ calculated in (3) is lower than the threshold calculated in (4), the affordance model prompts the SC to increase the node density in a certain area explained in Section III-A. The model sends the center of attention $\left(C_{a}\right)$ the radius size $\left(R_{a}\right)$ of nonhomogeneous vectors calculated as follows: $C_{a}=1 / N^{(\text {non-H) }} \sum_{i=1}^{i=N^{(\text {non-H) }}} h_{i}\left(\right.$ for $\left.\theta\left(N_{i}\right)>\Theta\right), R_{a}=$ $\sum_{i=1}^{i=N^{(\text {non-H) }}} \arg \max \left(h_{i}-C_{a}\right)$, where $\theta\left(N_{i}\right)$ is the angle between vector $N_{i}$ and the vertical vector. $N^{(\text {non-H) }}$ represents the number of nonhomogeneous normal vectors of nodes The attention area in the proposed model is limited to the pathway area of the robot movement

$$
\alpha=R_{a} / N^{(\text {non-H) }} .
$$

2) Representing Cortex and Movement-Related Processing Integration: The pathway process from the cortex to movement-related motor cortex shows how the affordances are represented to the motor cortex. The visual cortex model sends information about the detected object or obstacle (size and position), where its size and position are represented by parameter $C_{a}, R_{a}$ in Section III-B1. In our model, the nonhomogeneous vector in the attention area will be detected as the object or obstacle during movement. After that, the affordance perception will serve the possible action and safe area by detecting the normal vector to the vertical direction in the attention area.

\section{Movement-Related Command}

Locomotion involves two command pathways: one for gross movement mainly via the reticulospinal tract, and another for fine movement mainly via the corticospinal tract [39]. By taking advantage of this biological process, we build an efficient mechanism between external sensory information (cognition)

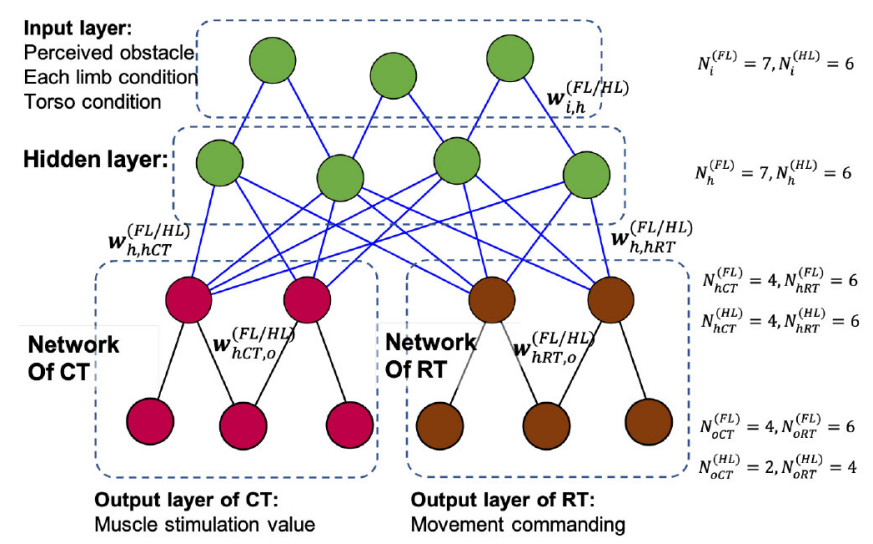

Fig. 4. Design of the movement command model integrates perception information into the locomotion generator model. The CT (corticospinal tract) network connects directly to the muscle reflex system. The RT (reticulospinal tract) network drives signal to the central pattern generator.

and action (muscle model). We separate the control pathway for gross movement (speed and direction) and fine movement (positioning and joint control). It will be effective when the system detects a sudden obstacle. When the system recognizes a safe or flat terrain, the model communicates movement speed and direction to the CPG model through the reticulospinal pathway. In other circumstances, when the system detects obstacles to avoid and changes the swing pattern, associated signals are sent through the corticospinal pathway. The model is represented by an artificial neural network that deals with both object detection and movement commands. The network structure is illustrated in Fig. 4. The main network is represented by green neurons and separated into the CT network representing the corticospinal tract and the RT network representing the reticulospinal tract. The input layer takes the perceived obstacle information (location, size), the state parameters of each limb (phase, joint angles, limb vector movement), and the torso's state parameters (tilt and speed). $\mathbf{w}^{(\mathrm{FL} / \mathrm{HL})}$ represents the synaptic weights and $N^{(\mathrm{FL} / \mathrm{HL})}$ represents the number of neurons in each layer.

When an obstacle is perceived during a limb's swing phase, the CT network is activated to overwrite the current muscle stimulation (MN), bypassing the CPG network. If the obstacle is perceived before the limb's swinging phase is activated, the RT network will be activated to send swing commands through the CPG level. The output of the CT network contains two parameters $\left(l_{C T, 1}, l_{C T, 2}\right)$ and four parameters $\left(l_{C T, 3}, l_{C T, 4}, l_{C T, 5}, l_{C T, 6}\right)$ transferred to the motoneuron of hindlimb and forelimb, respectively, explained in Section V. Then, the output of the RT network represented by $c_{\mathrm{RT}, \mathrm{LEG}, \mathrm{i}}$ parameters is transferred to PF neurons, explained in Section IV-B. There are four parameters influence to hindlimb leg and six parameters influence to forelimb leg.

\section{INTEGRATEd CPG MOdeL}

The CPG model represents the neuronal circuit in the spinal cord that generates basic rhythmic signals for locomotion. These signals can be generated without sensory feedback [12]. Our model is inspired by the single-rhythm generator proposed 


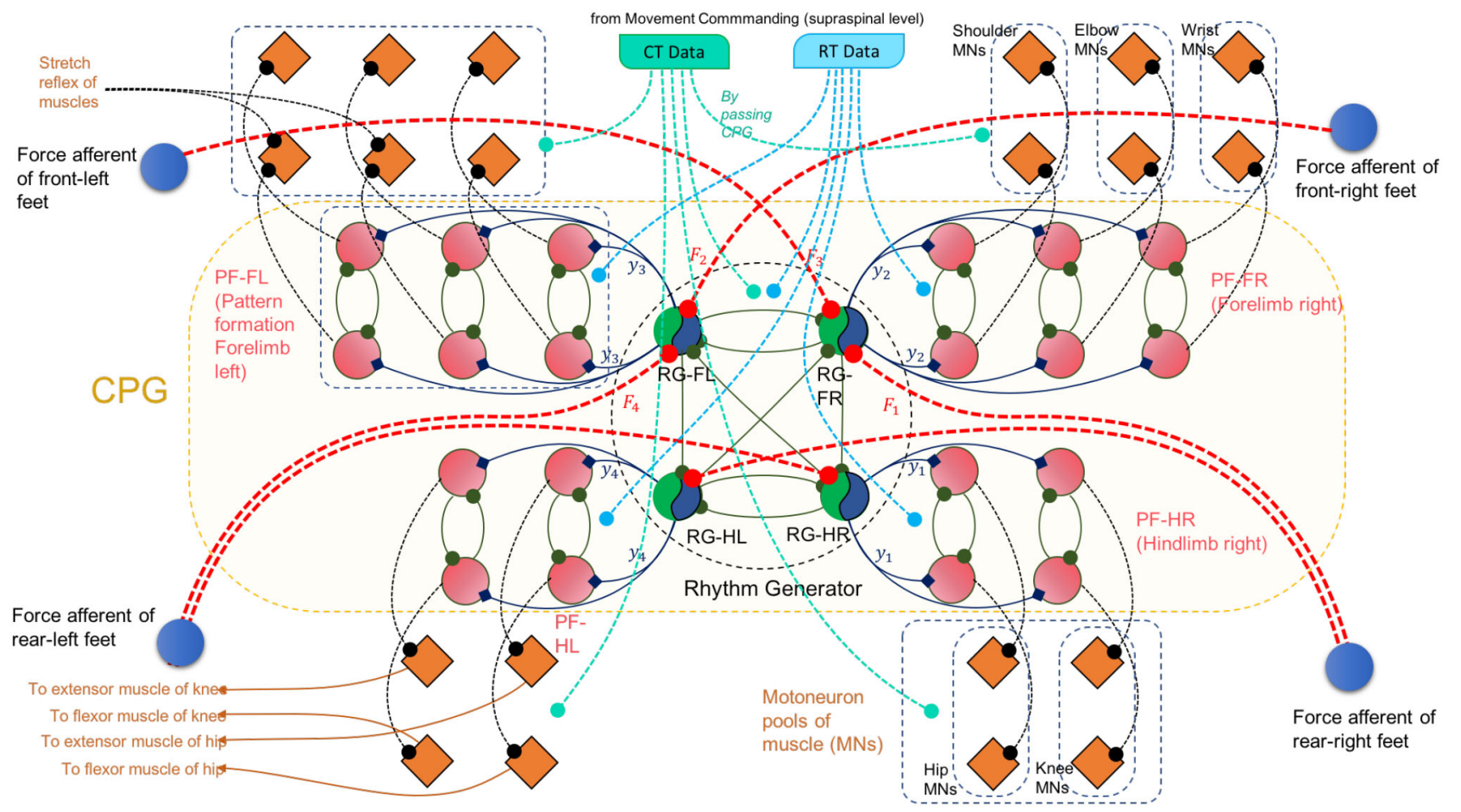

Fig. 5. Design of the two-layered CPG with distributed rhythm generator and PF.

by Rybak et al. [13], [31]. We improve the previous CPG model explained in [40] by adding input from the supraspinal level. We design a two-layered CPG model with a distributed single-rhythm generator and PF as illustrated in Fig. 5. We design a two-layered CPG model with a distributed singlerhythm generator and PF. Each rhythm generator represents one leg transfer of the rhythmic signal to the pattern generator. There are two PF neurons per joint.

\section{A. Rhythm Generator}

The rhythm generator produces an oscillating signal for PF. The signal should be able to dynamically change its rhythmic pattern. It uses the neural oscillator proposed by Matsuoka [41] to introduce reciprocal inhibition effects to the signal pattern [42]. It uses a second-order system of differential equations

$$
\begin{aligned}
\tau \frac{\mathrm{d}}{\mathrm{d} t} x_{i} & =\left(v_{i}-x_{i}-\sum_{j=1}^{n} w_{i j} y_{j}+\sum_{l=1}^{n} m_{i l} s_{l}-b v_{i}\right) \tau_{f} \\
T \frac{\mathrm{d}}{\mathrm{d} t} v_{i} & =\left(y_{i}-v_{i}\right) \tau_{f} \\
y_{i} & =\max \left(x_{i}, 0\right) .
\end{aligned}
$$

Variables $x_{i}, y_{i}$, and $v_{i}$ represent membrane potential, firing rate, and neuronal self-adaptation, respectively. The membrane potential is affected by excitatory tonic input $v_{i}$ and inhibitory input from other neurons $\sum_{j=1}^{n} w_{i j} y_{j}$, where $w_{i j}$ represents the synaptic weight between the $i$ th and $j$ th neurons. Membrane potential is also affected by afferent feedback and drive signals from the supraspinal level. The RG neurons are connected with force touch sensors in each foot and stance phase timing in each leg to synchronize the signal pattern. The firing rate of neuron $y$ will be transferred as the input to the PF model.

\section{B. Pattern Formation Model}

The PF model controls the signals sent to motoneurons for activating the muscles. The PF generates a firing-rate signal for the muscle, and can control the timing of the muscle activation in every cycle. In this model, we use a modified spiking neural network (SNN) [43] as the inner state of PF neurons to control the timing of the firing signal. The inner state of the PF neuron in the $i$ th joint $\left(\mathrm{PF}_{i}\right)$ is calculated as

$$
\begin{aligned}
\mathrm{PF}_{i, k}(t) & =e^{\left(\log (0.5) \times\left(\frac{\left|p f_{i}-\varphi\right|}{\left(\mu \times w \times c_{\mathrm{RT}, \mathrm{LEG}, \mathrm{i})}\right.}{ }^{3}\right)\right)} \\
p f_{i}(t) & =p f_{i}(t-1)+p_{i}
\end{aligned}
$$

where $c_{\text {RT,LEG,i }}$ represents the effect of the RT network to the $i$ th PF neuron of certain LEG. $\varphi$ represents the starting control calculated by $\varphi=\left(30-\phi_{i}^{(\mathrm{LEG})}\right) / 30$, and $w$ represents the time of activation signal calculated as $w=\psi_{i}^{(\mathrm{LEG})} / 50$. $\phi_{i}^{(\mathrm{LEG})}$ and $\psi_{i}^{(\mathrm{LEG})}$ are the parameters for controlling the swing activation and timing of the $i$ th PF neuron in certain LEG. The value of $\phi$ and $\psi$ will be optimized using a multiobjective evolutionary algorithm. $p_{i}$ is the spike signal calculated in the supplementary material -1 . The value $\mathrm{PF}_{i}$ is then transmitted to the muscle model to implement the actual swing.

\section{Musculoskeletal Model}

All mammals have a similar skeleton which includes both forelimb and hindlimb structures. In general, mamallian locomotion has both stance and swing phases. Several decades ago, some researchers proposed that rhythmic alternating contractions of muscles brought about cycling between the stance phase (extensor muscles) and the swing phase (flexor muscles). The rhythm can be generated without any sensory information from receptors in the skin, joints, and muscle [44], [45]. 


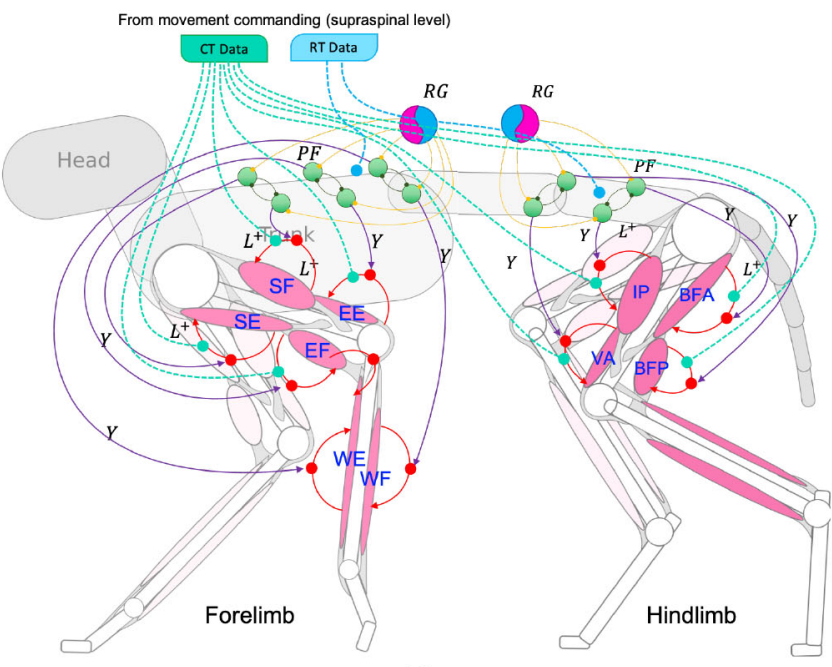

(a)

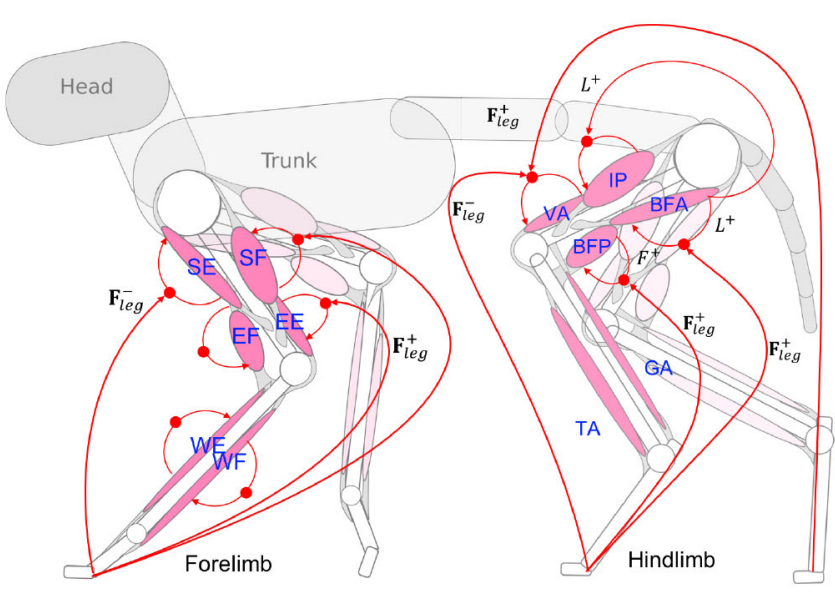

(b)

Fig. 6. Muscle reflex structure during its phase. (a) Muscle reflex structure in the swing phase. (b) Muscle reflex structure in the stance phase. $F_{\text {leg }}^{+}$and $F_{\text {leg }}^{-}$represent the positive and negative feedback of the force afferent.

Research on the stanceswing transition has yielded a substantial body of evidence that this transition is controlled by sensory signals from leg proprioceptors and has identified some of the receptors providing these signals [44].

The swing-to-stance transition unifies the two phases of stepping, rendering them inseparable [46], [47]. Based on this evidence, the swing-to-stance phase transition is activated automatically in response to stimuli from ground forces and hip positions. Thus, the swing-to-stance transition is the best phase in which to control the locomotion pattern. We control the swing stimulation using the signal pattern modulation from a neural oscillator proposed by Matsuoka. One CPG neuron represents one joint. The signal will stimulate the muscle to perform the swing phase. The muscle model is separated into two submodels for hindlimb and forelimb control depicted in Fig. 6. The detailed mathematical model can be seen in our previous work [48] and supplementary material-2.

From Fig. 6b, we can see the effect of CT directly to the muscle activation during the swing phase. It affects the value of the length offset in certain MN. Equations (10)-(13) show
MN for hindlimb during the swing phase. The effect of CT network to hindlimb is in $l_{\mathrm{CT}, 1}$ and $l_{\mathrm{CT}, 2}$ parameters

$$
\begin{aligned}
S_{\mathrm{BFA}}(t)= & S_{0, \mathrm{BFA}}-G_{\mathrm{BFA}}^{y} Y_{\mathrm{IP}} \\
& +G_{\mathrm{BFA}}^{l, S}\left(l_{\mathrm{CE}, \mathrm{BFA}}-l_{\mathrm{off}, \mathrm{BFA}}-l_{\mathrm{CT}, 1}\right) \\
S_{\mathrm{IP}}(t)= & S_{0, \mathrm{IP}}+G_{\mathrm{IP}}^{y} Y_{\mathrm{IP}}+G_{\mathrm{IP}}^{l, S}\left(l_{\mathrm{CE}, \mathrm{IP}}-l_{\mathrm{off}, \mathrm{IP}}-l_{\mathrm{CT}, 2}\right) \\
S_{\mathrm{VA}}(t)= & S_{0, \mathrm{VA}}-G_{\mathrm{VA}}^{Y} Y_{\mathrm{IP}}+C_{\mathrm{VA}} \\
S_{\mathrm{BFP}}(t)= & S_{0, \mathrm{BFP}}+G_{\mathrm{BFP}}^{Y} Y_{\mathrm{BFP}}-C_{\mathrm{BFP}}+G_{\mathrm{BFP}}^{F} F_{\text {leg }}
\end{aligned}
$$

Furthermore, (14)-(19) show $\mathrm{MN}$ for the forelimb during swing phase. The effect of the CT network to forelimb is in $l_{\mathrm{CT}, 3}$ to $l_{\mathrm{CT}, 6}$ parameters

$$
S_{\mathrm{SF}}(t)=S_{(0, \mathrm{SF}}+G_{\mathrm{SF}}^{Y} Y_{\mathrm{SF}}+G_{\mathrm{SF}}^{l}\left(l_{\mathrm{CE}, \mathrm{SF}}-l_{\mathrm{off}, \mathrm{SF}}-l_{\mathrm{CT}, 3}\right)
$$

$$
\begin{aligned}
S_{\mathrm{SE}}(t)= & S_{0, \mathrm{SE}}-G_{\mathrm{SE}}^{Y} Y_{\mathrm{SE}}+G_{\mathrm{SE}}^{l}\left(l_{\mathrm{CE}, \mathrm{SF}}-l_{\mathrm{off}, \mathrm{SF}}-l_{\mathrm{CT}, 4}\right) \\
S_{\mathrm{EE}}(t)= & S_{0, \mathrm{SE}}-G_{\mathrm{EE}}^{Y} Y_{\mathrm{EE}}+G_{\mathrm{EE}}^{l} \cdot Y_{\mathrm{EE}} \\
& \cdot\left(l_{\mathrm{EE}}-\left(-l_{\mathrm{off}, \mathrm{EF}}-l_{\mathrm{CT}, 5}\right)\right. \\
S_{\mathrm{EF}}(t)= & S_{0, \mathrm{EF}}-G_{\mathrm{EF}}^{Y}\left(1-Y_{\mathrm{EF}}\right)+G_{\mathrm{EF}}^{l} \cdot Y_{\mathrm{EF}} \\
& \cdot\left(l_{\mathrm{EF}}-l_{\mathrm{off}, \mathrm{EF}}-l_{\mathrm{CT}, 6}\right) \\
S_{\mathrm{WE}}(t)= & S_{0, \mathrm{WE}}+G_{\mathrm{WE}}^{Y} Y_{\mathrm{WE}} \\
S_{\mathrm{WF}}(t)= & S_{0, \mathrm{WF}}+G_{\mathrm{WF}}^{Y}\left(1-Y_{\mathrm{WF}}\right)
\end{aligned}
$$

In the robot implementation, we convert the muscle activation to joint angle by calculating the resulting muscle torque to drive the joint motor. The calculation of the torque can be seen in [49, Appendix II].

\section{EXPERIMENTAL RESULTS}

We implemented the proposed model in a cat-like robot simulation using open dynamics engine with its musculoskeletal model.

\section{A. CPG and Muscle Reflex Optimization}

We defined the intervals of the parameters empirically to specify the parameter search space for the optimization process. Furthermore, synaptic weights in the CPG model and muscle reflex parameters must be optimized to generate appropriate signal patterns and activation signals. We optimized 32 parameters representing $\mathrm{MN}$ and $\mathrm{PFs}$ (CPG) using a multiobjective evolutionary algorithm (NSGA-II) [50], where the fitness function is calculated as follows:

$$
\begin{aligned}
f_{1} & =\sqrt{\left(d_{l}-l_{\text {total }}\right)^{2}} \\
f_{2} & =\sum_{t=1}^{T} \sum_{\text {leg }}^{4} \sum_{m=1}^{N_{m}} S_{m}^{(l e g)}(t)^{[43]}
\end{aligned}
$$

where $f_{1}$ is the evaluation function for the distance traveled and $f_{2}$ is the total MN. In this optimization, we set the value of the desired distance traveled $\left(d_{l}\right)$ as $3 \mathrm{~m}$.

We trained 128 individuals through 200 generations, with crossover and mutation probability set at 0.3 . To evaluate the fitness, we ran the robot simulation through 1000 time 


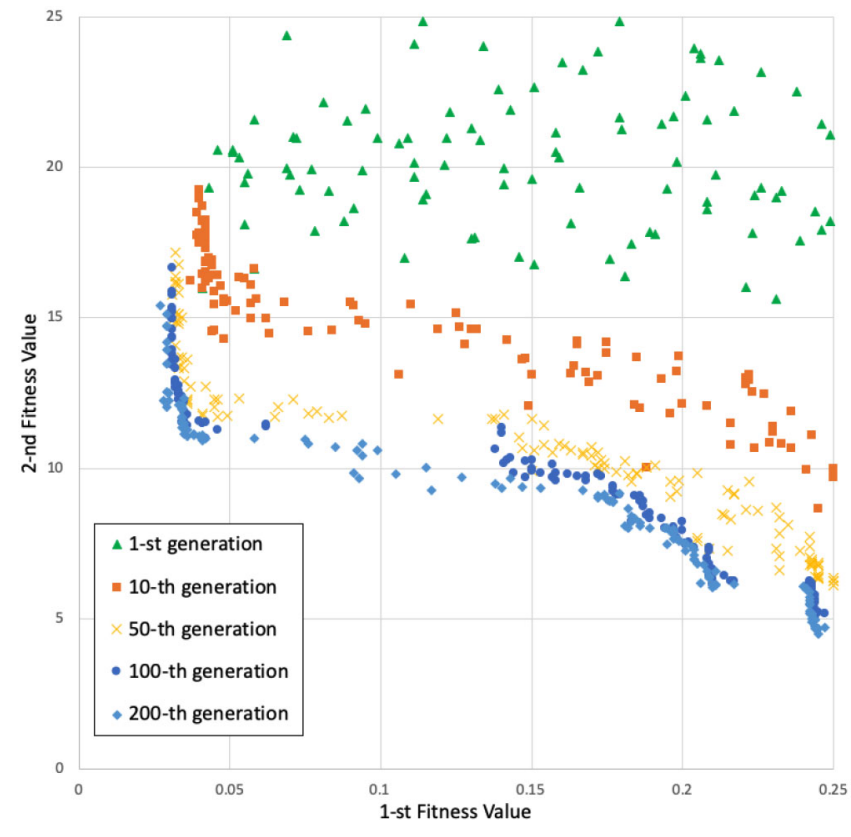

Fig. 7. Evolution of Pareto front in certain generation.

cycles $(5 \mathrm{~s})$. Then, we calculate the total distance traveled of the robot $\left(l_{\text {total }}\right)$. In the CPG structure, discrete signals from rhythm generation to the PF model are defined into a certain movement pattern. The RG structure is set similar to the previous model in [51]. As the optimization proceeded, the Pareto front evolved as shown in Fig. 7.

To coordinate the swing pattern of each limb, we optimized the RG structure with similar settings to our previous work [52]. We used a single-objective genetic algorithm (SSGA) as in our previous work [52]. In this optimization, we also optimized the influence of sensory feedback to the RG neurons in the CPG model. The resulting optimized parameters used for further robot performance in this article can be seen in Table I of the supplementary material. The signal output of the RG, PF, and MN in basic condition can be seen in the supplementary material-3.

\section{B. Optimization Process for the Movement Command Model}

Once the lower spinal level has been optimized, we can optimize the movement command model for controlling the appropriate signal for either the corticospinal pathway or reticulospinal pathway. We optimize the network for hindlimb and forelimb separately. Each network separates the optimized parameters into three groups: 1) A: weights parameter associated with green neurons only; 2) B: weights parameters associated with CT neurons; and 3) C: weights parameters associated with RT neurons. In the case when the fitness related to RT has a good result and the CT has a bad result, then the SSGA optimization evaluates the parameter of group B only. In the case when the fitness related to CT has a good result and RT has a bad result, then the SSGA optimization evaluates the parameter of group $\mathrm{C}$ only. The number of parameters optimized can be seen in Table I.
TABLE I

Total Number of Optimized PARAMETERS

\begin{tabular}{|c|c|c|c|c|c|c|c|}
\hline \multicolumn{2}{|c|}{ Group } & $\mathrm{A}$ & \multicolumn{2}{c|}{$\mathrm{B}$} & \multicolumn{2}{|c|}{$\mathrm{C}$} & Total \\
\hline \multicolumn{2}{|c|}{ Weight paramters } & $\mathbf{w}_{i, h}$ & $\mathbf{w}_{h, h C T}$ & $\mathbf{w}_{h C T, o}$ & $\mathbf{w}_{h, h R T}$ & $\mathbf{w}_{h R T, o}$ & \\
\hline \multirow{2}{*}{ Leg } & Forelimb & 49 & 28 & 16 & 42 & 36 & 171 \\
\cline { 2 - 7 } & Hindlimb & 36 & 24 & 8 & 36 & 24 & 128 \\
\hline
\end{tabular}

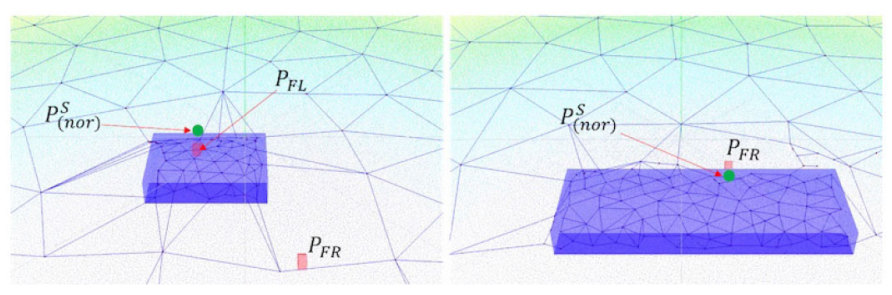

Fig. 8. Sample of the result of affordance detection. $P_{\mathrm{FL}}$ is the position of forelimb left foot and $P_{\mathrm{RF}}$ is the position of forelimb right foot.

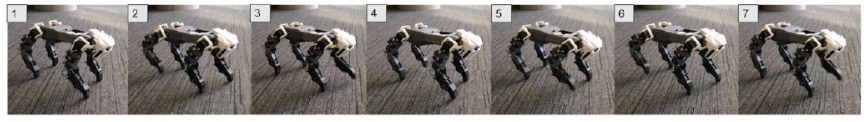

(a)

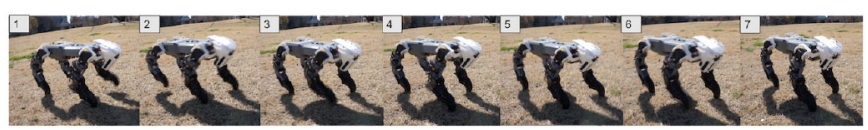

(b)

Fig. 9. Snapshot of the robot's performance of the locomotion generator model without supraspinal commands. (a) On flat terrain. (b) On natural grass.

Before that, we show the result of the attention control process and the object recognition as the input of the optimized model. A sample affordance detection with movement commanding result taken from the experiment in Section VII-B can be seen in Fig. 8.

1) Optimization for the CT Network: The CT network stimulates the muscular stretch reflex. We optimize the network to control the swing acceleration when facing a sudden obstacle. We evaluate the congruence of step length commands to the CT network and the resulting robot step length. In one evaluation, the robot performed 1000 time steps on flat terrain. We supply the network of $\mathrm{CT}$ with nine different command values, $I=\{-0.8,-0.6,-0.4,-0.2,0,0.2,0.4,0.6,0.8\}$ in the middle of the swing, when the feet are at or near their highest phase. We evaluate using the following measure:

$$
f=\sum_{i=1}^{9}\left(\frac{1}{1+\exp \left(s_{i}-s_{i+1}\right)}\right), s_{5}=S_{(\text {nor })}
$$

where $S_{(\text {nor) }}$ is the average step length in normal swing; $s_{i}$ is the step length of the $i$ th command. We assume the value of $s_{5}=0$ when $I_{5}=0$. We optimized using a single-objective evolutionary algorithm.

2) Optimization of the RT Network: The RT network will drive the CPG network, RG neurons, and/or PF neurons. We optimize the network to avoid obstacles with a certain height and a certain step length. We evaluate the congruence of step length and step height commands to the 


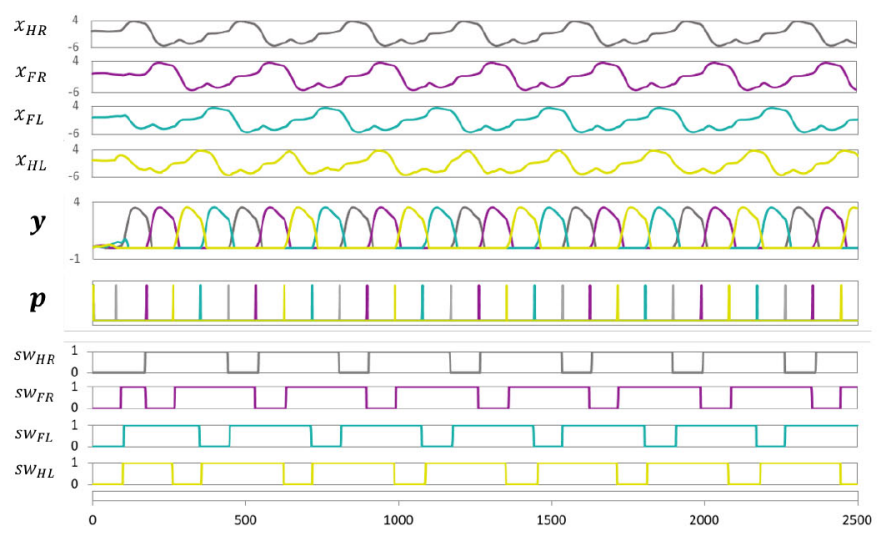

(a)

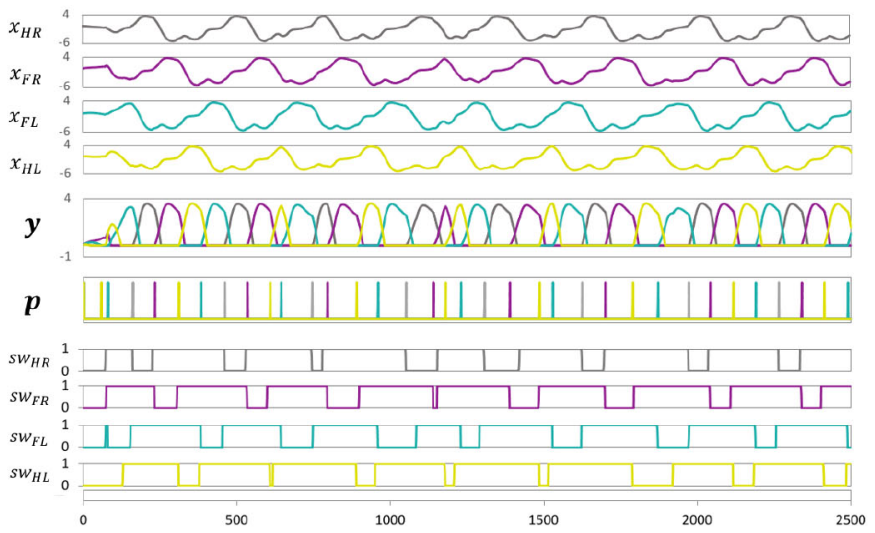

(b)

Fig. 10. Signal generated by components of the central pattern generator and internal sensory feedback. $x_{\mathrm{HR}}, x_{\mathrm{FR}}, x_{\mathrm{HL}}, x_{\mathrm{FL}}$ represent the inner state of rhythm generator neurons. $Y$ and $P$ are the output of RG neurons and PF neurons. $s w_{\mathrm{HL}}, s w_{\mathrm{FL}}, s w_{\mathrm{HR}}$, and $s w_{\mathrm{FR}}$ are the swing phase condition of the hindlimb-left, forelimb-left, hindlimb-right, and hindlimb-right, respectively. (a) On flat terrain and (b) on natural grass. The differences show the effect of sensory feedback to the rhythm generator during movement on natural terrain.

(a)

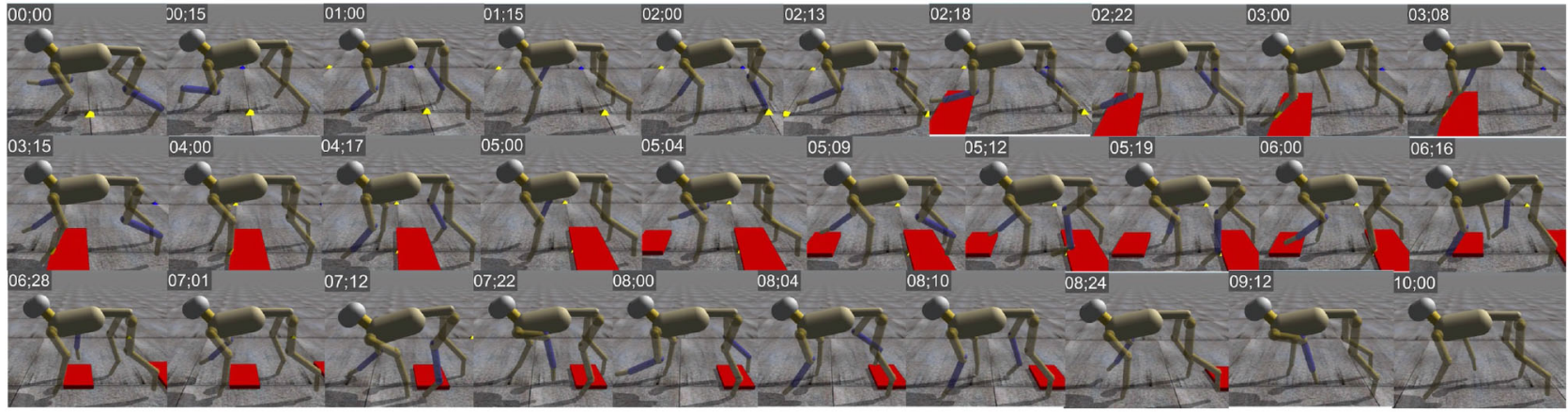

(b)
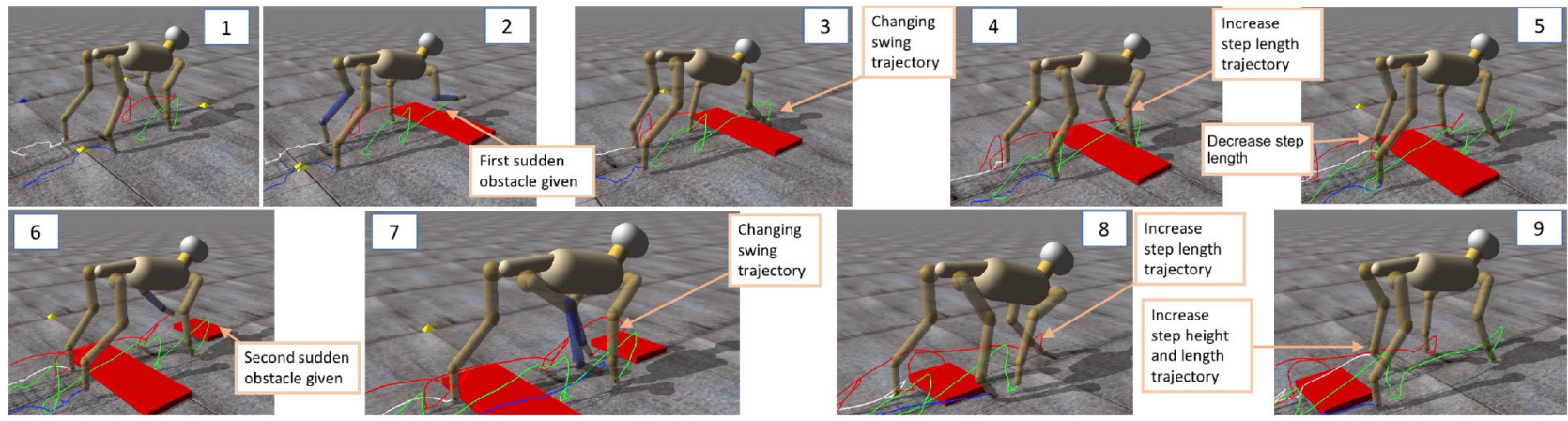

Fig. 11. Robot's performance in simulation. The detailed robot performance can be seen in the Supplementary video. (a) Time-series snapshot from side view. (b) Snapshot of the robot performance in certain condition from perspective view. 1) Robot performs in normal walking. 2) First sudden obstacle is given during left forelimb swing. 3) The left-forelimb changes the swinging pattern by reflex placing (see green line trajectory). 4) Right forelimb steps over the obstacle. 5) Right hindlimb decreased the step length because stepping over would have been too far. 6) Second sudden obstacle is given during the right forelimb swing phase. 7) Placing reflex to avoid the obstacle (see red line trajectory). 8) Right forelimb steps over the obstacle by increasing step length. 9) Right hindlimb increases step height and step length to avoid the second obstacle.

RT network with robot performance. In one evaluation, the robot performed 1000 time steps on flat terrain. We supply the network of RT with nine different command values, $I=\{-0.8,-0.6,-0.4,-0.2,0,0.2,0.4,0.6,0.8\}$ when the feet start swinging. The evaluation function is

$$
f=\sum_{i=1}^{9}\left(\frac{1}{1+\exp \left(s_{i}-s_{i+1}\right)}\right)+\sum_{i=1}^{9}\left(-h S_{i}\right) s_{5}=S_{(\text {nor })}
$$

where $h S_{i}$ is the height of step of the $i$ th command.

\section{Result of Robot Performance}

In order to test the effectiveness of the proposed model, the robot was assessed in several phases. First, we tested the synchronization of the internal sensory information, reflex system, and the CPG model without any commands from the supraspinal level. We tested the robot both in simulation and in a real implementation. After that, we examined the role of command signals from the supraspinal level. The robot performs on flat terrain and slope terrain and is given some sudden obstacles. 
(a)

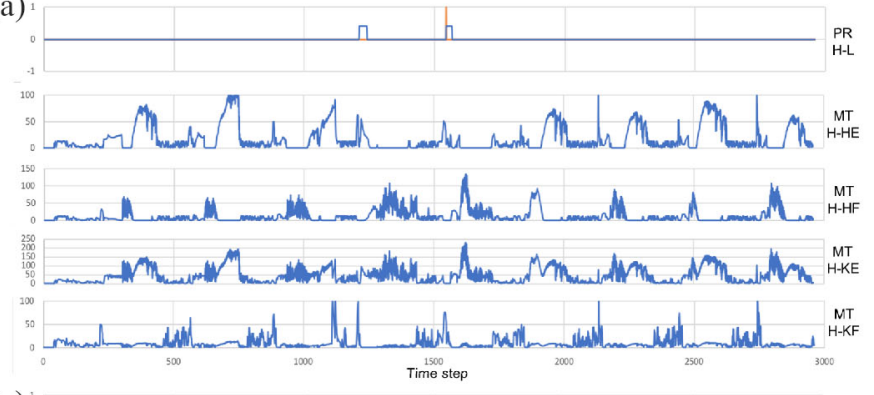

(c)

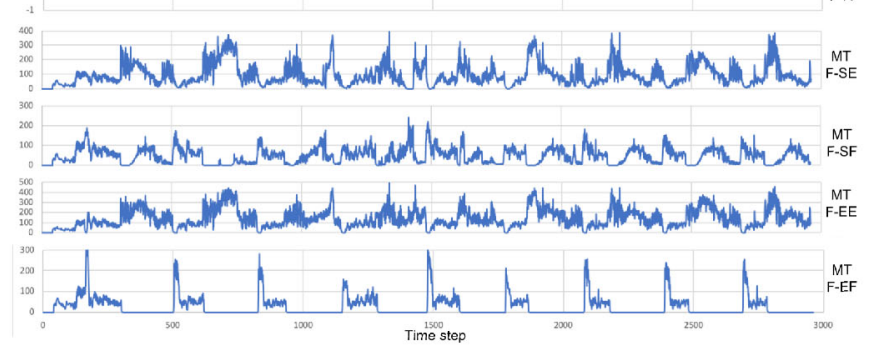

(b)

$P R \quad(d)$

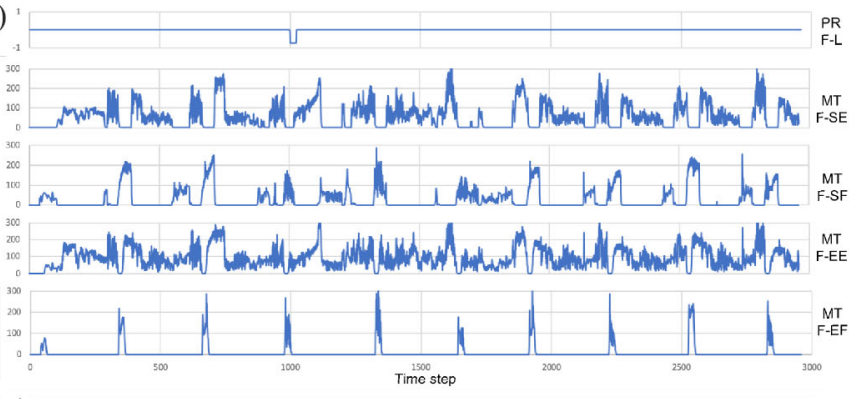

d)

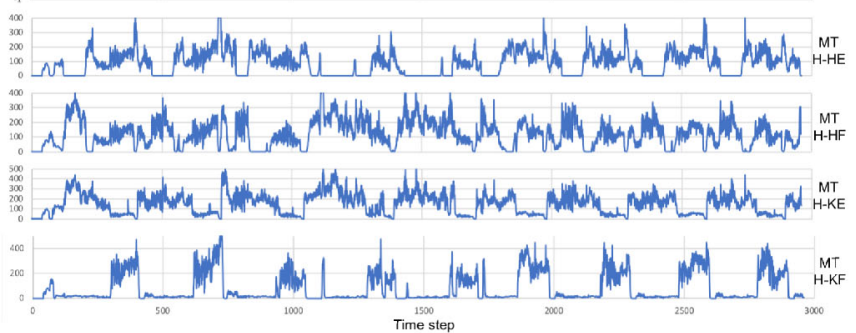

Fig. 12. Recorded torque when avoiding the sudden obstacle. (a) Right-hindlimb. (b) Right-forelimb. (c) Left-forelimb. (d) left-hindlimb.

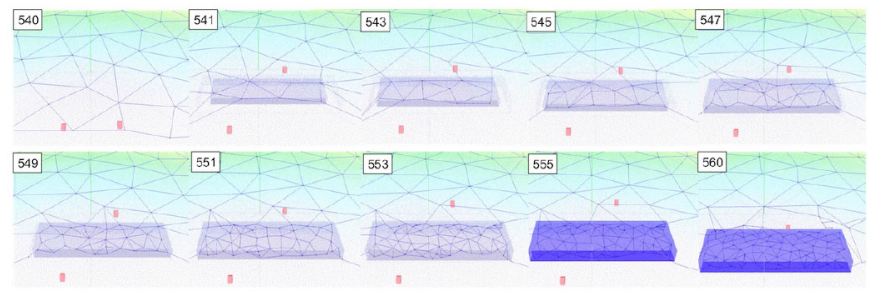

(a)

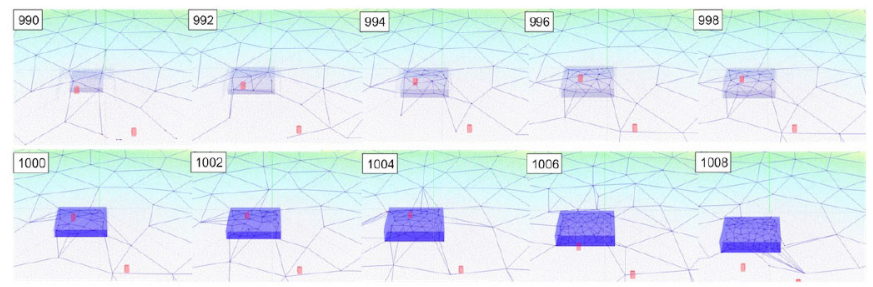

(b)

Fig. 13. Result of affordance detection. (a) First obstacle affordance detection. (b) Second obstacle affordance detection.

\section{A. Robot Performance Without Supraspinal Commands}

We tested on flat terrain in simulation and with a real robot. After that, we tested on natural terrain (grass) using the real quadruped robot. In order to evaluate individual legs, we set the CPG model to generate a walk-type gait. The snapshot of the robot performance can be seen in Fig. 9. We show the recorded signal of CPG and the locomotion event (touching condition) in Fig. 10 to evaluate the relationship between CPG and locomotion events. On flat terrain, the timing of swinging (detected from the touching condition) is similar in the whole performance. However, we can see many changes of swinging time when the robot performed on the natural terrain. The sensory feedback has an impact to the inner state of RG neuron. Then it changes the pattern of gait. Here, we can see the synchronization process between CPG and the locomotion event.

\section{B. Robot Performance When Suddenly Encountering Obstacle}

In order to show the effectiveness of the proposed model, we run the quadruped robot both in simulation and in real performance.

1) On Flat Terrain: In testing scenario, we twice put sudden obstacles, of different sizes, in the robot's way while it was walking. The obstacles were cuboids of size (length $\times$ width $\times$ height) $700 \mathrm{~mm} \times 230 \mathrm{~mm} \times 40 \mathrm{~mm}$, and $300 \mathrm{~mm} \times 200 \mathrm{~mm} \times 40 \mathrm{~mm}$. In the simulation, we put the first obstacle at time step equals 555 and the second obstacle at time step equals 1000 . The robot performance snapshot can be seen in Fig. 11. Fig. 11 illustrates the whole robot performance avoiding the obstacle from side view and snapshots of the robot's behavior when avoiding the obstacle under several conditions. To analyze the effect of muscle force, we show the recorded robot performance in Fig. 12.

In Fig. 11(b): 1) the robot moved in the normal condition from the starting condition; 2) the sudden obstacle is given at time step 541, when the left forelimb is swinging; and 3) the robot perceived the sudden obstacle at time step 555. It required 15 time samplings $(0.070 \mathrm{~s})$ for the cognitive model to perceive the model (see Fig. 13a, perceiving processing) and generate the overwriting $\mathrm{MN}$ signal from the CT network. It stimulated the shoulder extensor (SF) muscle to advance the limb (see Fig. 14 in fourth signal, for stimulation). 4) Since the existing obstacle did not affect the next foothold position, the right forelimb stepped over the obstacle without any overwriting command. 5) There was no possibility for the right hindlimb to step over the obstacle due to the limitation of the possible step length; the robot decreased the step length. 6) At time step 990, a second sudden obstacle was given when the right forelimb was in the swing phase. 7) At time step 1000, the robot perceived the obstacle (see Fig. $13 \mathrm{~b}$, perceiving processing) in 12 time steps $(0.05 \mathrm{~s})$. It stimulated the shoulder flexor (SF) muscle to draw back the 


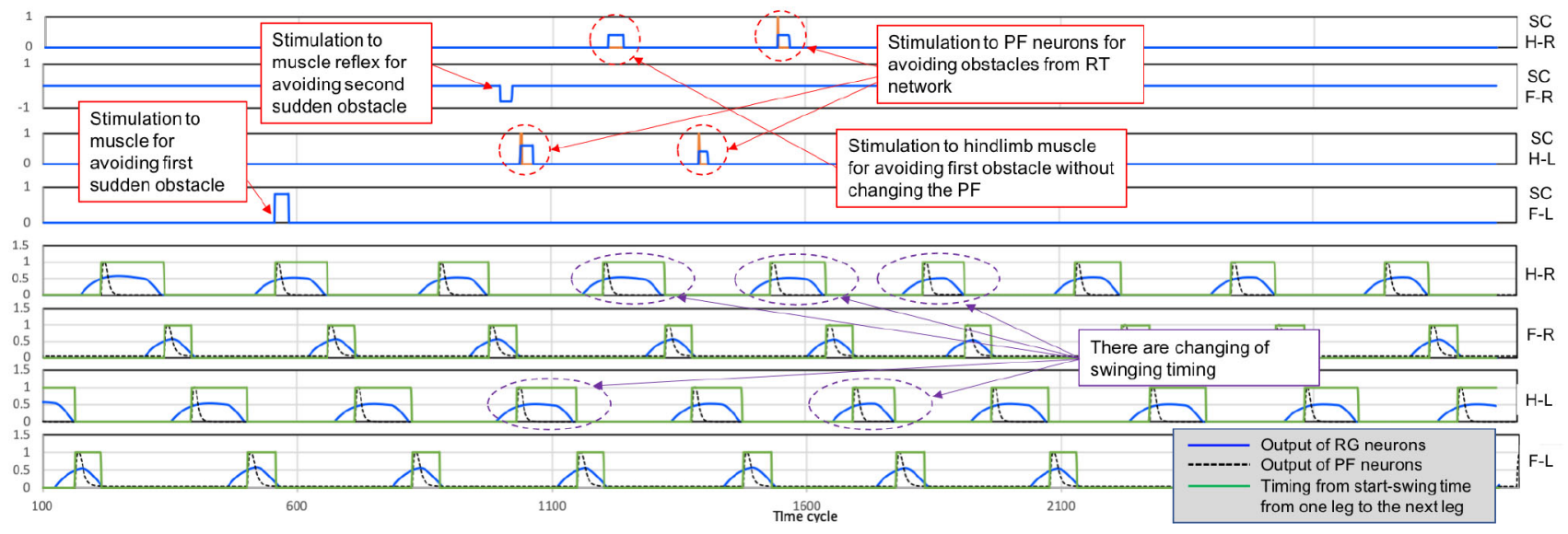

Fig. 14. Analysis of signal commanding and its effect on the CPG and movement timing.

limb (see Fig. 14 in second signal, for stimulation). 8) The right forelimb step length was increased by command from the RT network. 9) The right hindlimb received a command signal from the CT to change the swing pattern to avoid the obstacle by increasing step height and step length [see white line trajectory in Fig. 11(b)-9].

Furthermore, in order to evaluate the robustness of the proposed model, the robot performed 50 times, where in each trial, we put one sudden obstacle $(300 \mathrm{~mm} \times$ $200 \mathrm{~mm} \times 40 \mathrm{~mm}$ ) in a random position in front of the robot. The robot successfully avoided the obstacle 41 times, that is, in $82 \%$ of the trials.

2) On Sloped Terrain: The robot performed on sloped terrain with five obstacles which are listed as follows.

1) $700 \mathrm{~mm} \times 230 \mathrm{~mm} \times 40 \mathrm{~mm}$.

2) $300 \mathrm{~mm} \times 200 \mathrm{~mm} \times 40 \mathrm{~mm}$.

3) $500 \mathrm{~mm} \times 140 \mathrm{~mm} \times 45 \mathrm{~mm}$.

4) $200 \mathrm{~mm} \times 100 \mathrm{~mm} \times 50 \mathrm{~mm}$.

5) $600 \mathrm{~mm} \times 200 \mathrm{~mm} \times 45 \mathrm{~mm}$.

We placed the first and second sudden obstacles during uphill movement. After that, we put the third, fourth, and fifth during downhill movement. A sample snapshot of the robot's performance can be seen in Fig. 15 and a detailed video can be seen in the supplementary video.

Furthermore, to confirm the robustness on terrain at $20^{\circ}$ inclination, the robot performed 25 times uphill and 25 times downhill terrain. We conducted similar trials to evaluate the robustness on flat terrain. The robot successfully avoided the obstacle 17 times uphill (68\% success) and 22 times (88\% success) downhill. The difference is due to the fact that, when walking uphill, the robot needs a higher swing to avoid the obstacle than when walking on the flat or downhill.

3) Real Robot Implementation: After successfully implementing the simulation, we tested the proposed model in a medium-size cat-like robot. The robot has several internal sensors, such as IMU sensors and force sensors in each leg, and an external time-of-flight sensor. The robot uses an Intel core i5 CPU without any GPU. Joints have an angle-based actuator. Therefore, we converted the value of muscle force to joint angle. In the first performance, we placed an obstacle before the robot started. In the second performance, we put the sudden obstacle exactly in front of the robot three times. In the third performance, we put a bigger obstacle, a further distance in front of the robot. The result can be seen in Fig. 16. The robot successfully avoided the obstacle in all three performances. The video demonstration can be seen in the supplementary material.

\section{DISCUSSION}

We proposed a model for neurocognitive locomotion in a neuromusculoskeletal model. Our model integrates the external sensory information process with the locomotion generator. In this article, we focus on modeling the placing reflex to generate behavior for avoiding sudden obstacles in a musclebased model. Our results suggest that it is worth viewing the cognitive and MN models as a single, unified system, rather than separately

We began this article by analyzing human and animal locomotion from a physiological view point that showed the role of cognition in locomotion [8]-[10]. Our viewpoint is also supported by neuroactivity experiments that demonstrate the contribution of supraspinal activity to motor neuron activation. Specifically, analysis of the corticospinal tract has shown that there is a low activity of CT in the uniform area or on flat paths but, when obstacles stand in the way, CT activity is higher especially in the swing phase [16]. Thus, limb control to avoid obstacles requires cognitive processing of external sensory information [8]. From these foundations, we sought to mimic nature as we developed a locomotion model that included supraspinal commands to the locomotion generator. We implemented a descending process from the external sensory process to limb movement generator. We applied the proposed model to a quadruped robot.

We implemented an attention model mimicking that of humans. The robot's external input uses point cloud data instead of visual information, however, this choice of representation does not influence the attention mechanism's implementation. Attention was represented using nodal density in a topological map structure, as had been successfully implemented in our previous research [35]. In this article, we built a dynamic attention model inspired by visual processing 


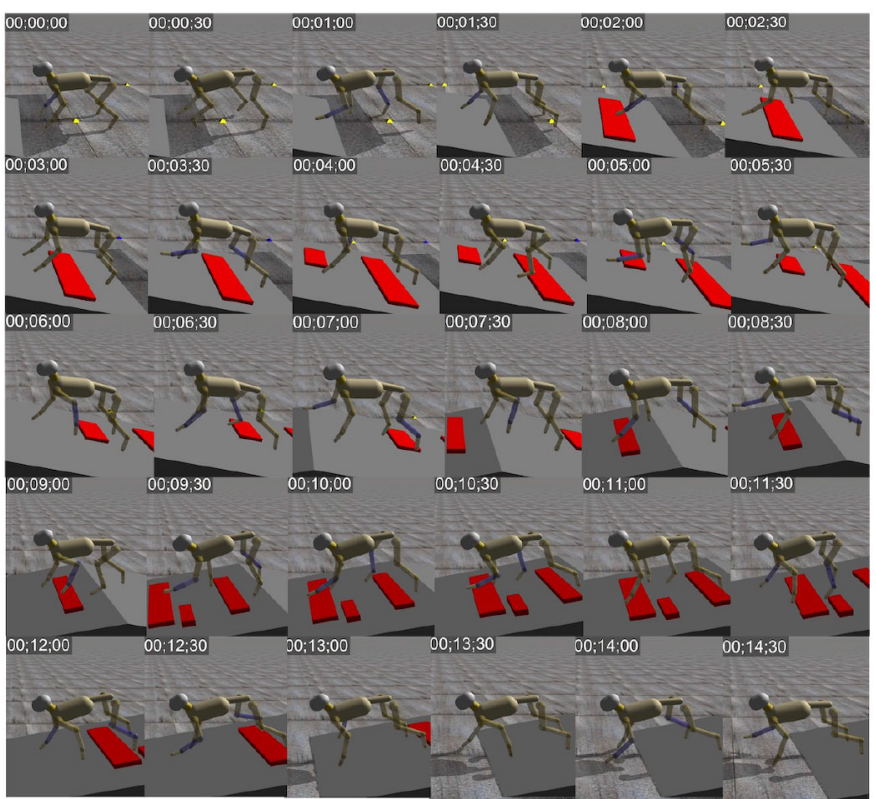

Fig. 15. Time-series snapshot of the simulated robot performance in slope terrain with sudden obstacles given. The detailed robot performance can be seen in the supplementary video.

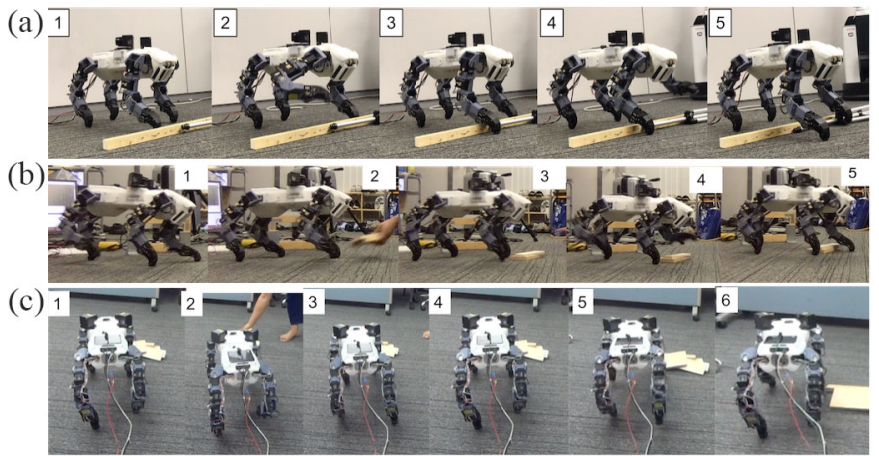

Fig. 16. Snapshot of real robot performance (a) with obstacle in front of the robot, (b) with sudden obstacle exactly in front of the robot, and (c) with bigger obstacle a little bit far in front of the robot.

processes in nature. Topological structure has a low density for walking across flat, clear surfaces, and the density increases when there are obstacles. This mechanism is computationally efficient, with one process costing $0.00314 \mathrm{~s}$. As the number of obstacles increased, the processing cost also increased. Our mechanism is, in this way, similar to the human visual process: when there are a lot of obstacles, cortex activity also increases [53].

The topological information is then processed to detect object affordances. In our proposed system, we do not need to recognize objects in detail: our model successfully avoided the obstacles without detailed obstacle recognition. In the experiments, we assume that the objects should be avoided. However, the way to avoid depends on the object affordance detection. The robot avoids the obstacle by shortening the swing, changing the swing direction, lifting the swing, and changing the walking direction. Our results show that the locations and sizes of suddenly appearing obstacles can be recognized in 0.07 and $0.06 \mathrm{~s}$ for the first and second object, respectively. Our results also show an interesting integration of attention control, object affordance recognition, and movement commanding. In the case of unclear recognition, the object recognition stimulated increased attention to the suspected area. Once the object is recognized, the object affordance detector provides for an appropriate motor response.

The command process successfully commanded the forelimb to avoid sudden obstacles by stimulation through the CT network. While avoiding the obstacle, changes to the RG and PF signals modify the timing of the swing phase. In the robustness evaluation, the robot had a success rate of $82 \%$ on flat terrain, $68 \%$ on uphill terrain, and $88 \%$ on downhill terrain. The robot could easily avoid the obstacle when going downhill because it does not need much higher swinging movement. Vice versa, on the uphill terrain, the robot needs to swing its legs higher. A large experimental trial would be required to investigate the applicability of the proposed model. However, the present experimental results show the effectiveness and the importance of integrating cognition with the locomotion generator. For future work, the stability of the system should be improved in order to increase the success rates.

We have proved and showed that our model combines the cognitive information and musculoskeletal model by integrating exteroceptive sensory information and low-level control muscle control in Section VII. The system can therefore respond to environmental changes in every time cycle. Existing systems that integrate perception and locomotion mostly deal with foothold planning, which limits adaptation to the much longer timescale of whole footsteps [54]-[61]. While [62] and [63] dealt with real-time obstacle avoidance, their system is unable to cope with obstacles that suddenly block an alreadyswinging limb. Furthermore, the bioinspired-based locomotion model's short-term adaptation controllers consider only internal sensory information, such as from posture, touching, and inertial information. Thus, the robot cannot respond to an obstacle before touching it. Our proposed system has the advantage in controlling the locomotion in every time cycle, which has been proved in several experiments avoiding unknown sudden obstacles.

Generally, our neurocognitive locomotion model shows a way to integrate neuromusculoskeletal models with cognitive models for achieving dynamic locomotion. Such integration may have a sizeable impact not only in robotics but also in medical applications, such as in the development of active prosthetic limbs. Active prosthetics have been shown to increase patient confidence during ambulation [64]. By monitoring eye movement signals from the posterior parietal reach region, [65], [66] and using visual priority map [67], the neurocognitive locomotion model can be integrated into knee prosthetics to actively avoid sudden obstacles or suddenly changing swing patterns.

\section{REFERENCES}

[1] Y. Wang, X. Xue, and B. Chen, "Matsuoka's CPG with desired rhythmic signals for adaptive walking of humanoid robots," IEEE Trans. Cybern., vol. 50, no. 2, pp. 613-626, Feb. 2020. 
[2] X. Xiong, F. Wörgötter, and P. Manoonpong, "Adaptive and energy efficient walking in a hexapod robot under neuromechanical control and sensorimotor learning," IEEE Trans. Cybern., vol. 46, no. 11, pp. 2521-2534, Nov. 2016.

[3] A. A. Saputra, J. Botzheim, I. A. Sulistijono, and N. Kubota, "Biologically inspired control system for 3-D locomotion of a humanoid biped robot," IEEE Trans. Syst., Man, Cybern., Syst., vol. 46, no. 7, pp. 898-911, Jul. 2016

[4] T. Geijtrnbeek, M. Van De Panne, and A. F. Van Der Stappen, "Flexible muscle-based locomotion for bipedal creatures," ACM Trans. Graphics, vol. 32, no. 6, p. 206, 2013.

[5] H. Witte, M. S. Fischer, H. Preuschoft, D. Voges, C. Schilling, and A. J. Ijspeert, "Quadruped locomotion," Living Machines: A Handbook of Research in Biomimetic and Biohybrid Systems. Oxford, U.K.: Oxford Univ. Press, 2018, p. 289.

[6] A. J. Ijspeert, "Central pattern generators for locomotion control in animals and robots: A review," Neural Netw., vol. 21, no. 4, pp. 642-653, 2008.

[7] A. J. Ijspeert, "Biorobotics: Using robots to emulate and investigate agile locomotion," Science, vol. 346, no. 6206, pp. 196-203, 2014.

[8] A. E. Patla, "Understanding the roles of vision in the control of human locomotion," Gait Posture, vol. 5, no. 1, pp. 54-69, 1997.

[9] D. Logan et al., "The many roles of vision during walking," Exp. Brain Res., vol. 206, no. 3, pp. 337-350, 2010.

[10] M. Laurent and J. A. Thomson, "The role of visual information in control of a constrained locomotor task," J. Motor Behav., vol. 20, no. 1, pp. 17-37, 1988.

[11] T. G. Brown, "On the nature of the fundamental activity of the nervous centres; together with an analysis of the conditioning of rhythmic activity in progression, and a theory of the evolution of function in the nervous system," J. Physiol., vol. 48, no. 1, pp. 18-46, 1914.

[12] G. N. Orlovskiǔ, T. Deliagina, and S. Grillner, Neuronal Control of Locomotion: From Mollusc to Man. Oxford, U.K.: Oxford Univ. Press, 1999.

[13] I. A. Rybak, K. Stecina, N. A. Shevtsova, and D. A. McCrea, "Modelling spinal circuitry involved in locomotor pattern generation: Insights from the effects of afferent stimulation," J. Physiol., vol. 577, no. 2, pp. 641-658, 2006

[14] K. Pearson, Ö. Ekeberg, and A. Büschges, "Assessing sensory function in locomotor systems using neuro-mechanical simulations," Trends Neurosci., vol. 29, no. 11, pp. 625-631, 2006.

[15] J. J. Feher, Quantitative Human Physiology: An Introduction. New York, NY, USA: Academic, 2017.

[16] T. Drew, W. Jiang, B. Kably, and S. Lavoie, "Role of the motor cortex in the control of visually triggered gait modifications," Can. J. Physiol. Pharmacol., vol. 74, no. 4, pp. 426-442, 1996.

[17] S. Rossignol, R. Dubuc, and J.-P. Gossard, "Dynamic sensorimotor interactions in locomotion," Physiol. Rev., vol. 86, no. 1, pp. 89-154, 2006.

[18] D. M. Armstrong and T. Drew, "Discharges of pyramidal tract and other motor cortical neurones during locomotion in the cat," J. Physiol., vol. 346, no. 1, pp. 471-495, 1984.

[19] D. M. Armstrong and T. Drew, "Locomotor-related neuronal discharges in cat motor cortex compared with peripheral receptive fields and evoked movements," J. Physiol., vol. 346, no. 1, pp. 497-517, 1984.

[20] D. M. Armstrong and T. Drew, "Topographical localization in the motor cortex of the cat for somatic afferent responses and evoked movements," J. Physiol., vol. 350, no. 1, pp. 33-54, 1984.

[21] I. N. Beloozerova and M. G. Sirota, "The role of the motor cortex in the control of accuracy of locomotor movements in the cat," J. Physiol., vol. 461 , no. 1 , pp. $1-25,1993$.

[22] I. N. Beloozerova, M. G. Sirota, H. A. Swadlow, G. N. Orlovsky, L. B. Popova, and T. G. Deliagina, "Activity of different classes of neurons of the motor cortex during postural corrections," J. Neurosci., vol. 23, no. 21, pp. 7844-7853, 2003.

[23] W. Widajewicz, B. Kably, and T. Drew, "Motor cortical activity during voluntary gait modifications in the cat. II. Cells related to the hindlimbs," J. Neurophysiol., vol. 72, no. 5, pp. 2070-2089, 1994.

[24] S. Lavoie and T. Drew, "Discharge characteristics of neurons in the red nucleus during voluntary gait modifications: A comparison with the motor cortex," J. Neurophysiol., vol. 88, no. 4, pp. 1791-1814, 2002.

[25] M.-J. Rho, S. Lavoie, and T. Drew, "Effects of red nucleus microstimulation on the locomotor pattern and timing in the intact cat: A comparison with the motor cortex," J. Neurophysiol., vol. 81, no. 5, pp. 2297-2315, 1999.

[26] C. T. Leonard, The Neuroscience of Human Movement. St. Louis, MO, USA: Mosby, 1998.
[27] D. C. Van Essen, C. H. Anderson, and D. J. Felleman, "Information processing in the primate visual system: An integrated systems perspective," Science, vol. 255, no. 5043, pp. 419-423, 1992.

[28] K. M. Chu, S. H. Seto, I. N. Beloozerova, and V. Marlinski, "Strategies for obstacle avoidance during walking in the cat," J. Neurophysiol., vol. 118, no. 2, pp. 817-831, 2017.

[29] B. R. Fajen and W. H. Warren, "Behavioral dynamics of steering, obstable avoidance, and route selection." J. Exp. Psychol. Human Perception Perform., vol. 29, no. 2, p. 343, 2003.

[30] D. A. McCrea and I. A. Rybak, "Organization of mammalian locomotor rhythm and pattern generation," Brain Res. Rev., vol. 57, no. 1, pp. 134-146, 2008.

[31] I. A. Rybak, N. A. Shevtsova, M. Lafreniere-Roula, and D. A. McCrea, "Modelling spinal circuitry involved in locomotor pattern generation: Insights from deletions during fictive locomotion," J. Physiol., vol. 577, no. 2, pp. 617-639, 2006.

[32] G. Orlovsky, "The effect of different descending systems on flexor and extensor activity during locomotion," Brain Res., vol. 40, no. 2, pp. 359-372, 1972.

[33] M. A. Goodale and A. D. Milner, "Separate visual pathways for perception and action," Trends Neurosci., vol. 15, no. 1, pp. 20-25, 1992.

[34] A. A. Saputra, Y. Toda, N. Takesue, and N. Kubota, "A novel capabilities of quadruped robot moving through vertical ladder without handrail support," in Proc. IEEE/RSJ Int. Conf. Intell. Robots Syst., Nov. 2019, pp. $1448-1453$.

[35] A. A. Saputra, W. H. Chin, Y. Toda, N. Takesue, and N. Kubota, "Dynamic density topological structure generation for real-time ladder affordance detection," in Proc. IEEE/RSJ Int. Conf. Intell. Robots Syst., Nov. 2019, pp. 3439-3444.

[36] A. A. Saputra, C. W. Hong, and N. Kubota, "Real-time grasp affordance detection of unknown object for robot-human interaction," in Proc. IEEE Int. Conf. Syst. Man Cybern., Oct. 2019, pp. 3093-3098.

[37] J. J. Gibson, "The theory of affordances," in Perceiving, Acting, and Knowing: Toward an Ecological Psychology, R. Shaw and J. Bransford, Eds. Hillsdale, NJ, USA: Lawrence Erlbaum Assoc., 1977, p. 6782.

[38] M. T. Turvey, "Affordances and prospective control: An outline of the ontology," Ecol. Psychol., vol. 4, no. 3, pp. 173-187, 1992.

[39] S. N. Baker, "The primate reticulospinal tract, hand function and functional recovery," J. Physiol., vol. 589, no. 23, pp. 5603-5612, 2011.

[40] A. A. Saputra, A. J. Ijspeert, and N. Kubota, "A neural primitive model with sensorimotor coordination for dynamic quadruped locomotion with malfunction compensation," in Proc. IEEE/RSJ Int. Conf. Intell. Robots Syst. (IROS), 2020, pp. 3783-3788.

[41] K. Matsuoka, "Sustained oscillations generated by mutually inhibiting neurons with adaptation," Biol. Cybern., vol. 52, pp. 367-376, Oct. 1985.

[42] K. Matsuoka, "Mechanisms of frequency and pattern control in the neural rhythm generators," Biol. Cybern., vol. 56, pp. 345-353, Jul. 1987.

[43] W. Maass, "Networks of spiking neurons: The third generation of neural network models," Neural Netw., vol. 10, no. 9, pp. 1659-1671, 1997.

[44] K. Pearson, "Role of sensory feedback in the control of stance duration in walking cats," Brain Res. Rev., vol. 57, no. 1, pp. 222-227, 2008.

[45] T. G. Brown, "The intrinsic factors in the act of progression in the mammal," Proc. Roy. Soc. London B, vol. 84, no. 572, pp. 308-319, 1911.

[46] D. A. McVea, J. M. Donelan, A. Tachibana, and K. G. Pearson, "A role for hip position in initiating the swing-to-stance transition in walking cats," J. Neurophysiol., vol. 94, no. 5, pp. 3497-3508, 2005.

[47] T. Lam and K. G. Pearson, "Proprioceptive modulation of hip flexor activity during the swing phase of locomotion in decerebrate cats," $J$. Neurophysiol., vol. 86, no. 3, pp. 1321-1332, 2001.

[48] A. A. Saputra, W. H. Chin, A. J. Ijspeert, and N. Kubota, "A musclereflex model of forelimb and hindlimb of felidae family of animal with dynamic pattern formation stimuli," in Proc. Int. Joint Conf. Neural Netw., Jul. 2020, pp. 1-8.

[49] H. Geyer and H. Herr, "A muscle-reflex model that encodes principles of legged mechanics produces human walking dynamics and muscle activities," IEEE Trans. Neural Syst. Rehabil. Eng., vol. 18, no. 3, pp. 263-273, Jun. 2010.

[50] K. Deb, A. Pratap, S. Agarwal, and T. Meyarivan, "A fast and elitist multiobjective genetic algorithm: NSGA-II," IEEE Trans. Evol. Comput., vol. 6, no. 2, pp. 182-197, Apr. 2002.

[51] A. A. Saputra, N. N. W. Tay, Y. Toda, J. Botzheim, and N. Kubota, "Bézier curve model for efficient bio-inspired locomotion of low cost four legged robot," in Proc. IEEE/RSJ Int. Conf. Intell. Robots Syst., 2016, pp. 4443-4448. 
[52] A. A. Saputra and N. Kubota, "Synthesis of neural oscillator based dynamic rhythmic generation in quadruped robot locomotion," in Proc. IEEE Int. Electron. Symp. Knowl. Creation Intell. Comput., 2018, pp. $184-191$.

[53] S. R. Williams and L. N. Fletcher, "A dendritic substrate for the cholinergic control of neocortical output neurons," Neuron, vol. 101, no. 3, pp. 486-499, 2019.

[54] I. Havoutis, J. Ortiz, S. Bazeille, V. Barasuol, C. Semini, and D. G. Caldwell, "Onboard perception-based trotting and crawling with the hydraulic quadruped robot (HyQ)," in Proc. IEEE/RSJ Int. Conf. Intell. Robots Syst., 2013, pp. 6052-6057.

[55] H. W. Park, P. M. Wensing, and S. Kim, "Online planning for autonomous running jumps over obstacles in high-speed quadrupeds," in Proc. Robot. Sci. Syst. Conf., 2015, pp. 1-9.

[56] A. W. Winkler, C. Mastalli, I. Havoutis, M. Focchi, D. G. Caldwell, and C. Semini, "Planning and execution of dynamic whole-body locomotion for a hydraulic quadruped on challenging terrain," in Proc. IEEE Int. Conf. Robot. Autom., 2015, pp. 5148-5154.

[57] S. Kuindersma et al., "Optimization-based locomotion planning, estimation, and control design for the atlas humanoid robot," Auton. Robots, vol. 40, no. 3, pp. 429-455, 2016.

[58] R. Deits and R. Tedrake, "Footstep planning on uneven terrain with mixed-integer convex optimization," in Proc. IEEE-RAS Int. Conf. Humanoid Robots, 2014, pp. 279-286.

[59] R. J. Griffin, G. Wiedebach, S. McCrory, S. Bertrand, I. Lee, and J. Pratt, "Footstep planning for autonomous walking over rough terrain," 2019. [Online]. Available: arXiv:1907.08673.

[60] Y.-D. Hong and B. Lee, "Real-time feasible footstep planning for bipedal robots in three-dimensional environments using particle swarm optimization," IEEE/ASME Trans. Mechatron., vol. 25, no. 1 , pp. 429-437, Feb. 2019.

[61] M. Geisert, T. Yates, A. Orgen, P. Fernbach, and I. Havoutis, "Contact planning for the anymal quadruped robot using an acyclic reachabilitybased planner," in Proc. Аnnu. Conf. Auton. Robotic Syst., 2019, pp. 275-287.

[62] D. Wahrmann et al., "Vision-based 3D modeling of unknown dynamic environments for real-time humanoid navigation," Int. J. Humanoid Robot., vol. 16, no. 1, 2019, Art. no. 1950002.

[63] O. Villarreal et al., "Fast and continuous foothold adaptation for dynamic locomotion through CNNs," IEEE Robot. Autom. Lett., vol. 4, no. 2, pp. 2140-2147, Apr. 2019

[64] A. B. Sawers and B. J. Hafner, "Outcomes associated with the use of microprocessor-controlled prosthetic knees among individuals with unilateral transfemoral limb loss: A systematic review," J. Prosthetics Orthotics, vol. 25, no. 4S, pp. 4-40, 2013.

[65] S. Musallam, B. Corneil, B. Greger, H. Scherberger, and R. A. Andersen, "Cognitive control signals for neural prosthetics," Science, vol. 305, no. 5681, pp. 258-262, 2004

[66] R. Andersen, J. Burdick, S. Musallam, B. Pesaran, and J. Cham, "Cognitive neural prosthetics," Trends Cogn. Sci., vol. 8, no. 11, pp. 486-493, 2004.

[67] N. Anantrasirichai, K. A. Daniels, J. F. Burn, I. D. Gilchrist, and D. R. Bull, "Fixation prediction and visual priority maps for biped locomotion," IEEE Trans. Cybern., vol. 48, no. 8, pp. 2294-2306, Aug. 2018.

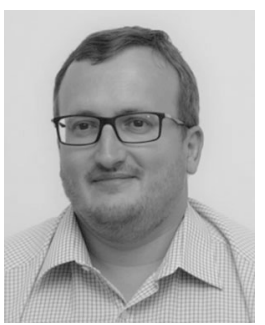

János Botzheim (Senior Member, IEEE) received the M.Sc. and Ph.D. degrees in computer science from the Budapest University of Technology and Economics, Budapest, Hungary, in 2001 and 2008, respectively.

He is an Associate Professor with the Department of Mechatronics, Optics and Mechanical Engineering Informatics, Budapest University of Technology and Economics. His research interests are computational intelligence and cognitive robotics.

Dr. Botzheim is a member of several scientific societies, such as the John von Neumann Computer Science Society and Hungarian Fuzzy Association.

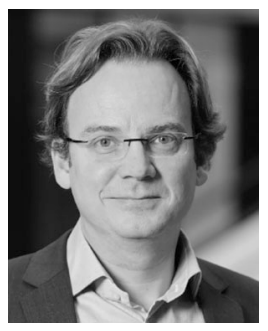

Auke Jan Ijspeert (Fellow, IEEE) received the B.Sc./M.Sc. degrees in physics from the Ecole Polytechnique Féderalé de Lausanne (EPFL), Lausanne, Switzerland, in 1995, and the Ph.D. degree in artificial intelligence from the University of Edinburgh, Edinburgh, U.K., in 1999.

He has been a Professor with EPFL since 2002, where he is the head of Biorobotics Laboratory. $\mathrm{He}$ is also investigating how to assist people with limited mobility using exoskeletons and assistive furniture. His research interests are at the intersection between robotics, computational neuroscience, nonlinear dynamical systems, and applied machine learning. He is interested in using numerical simulations and robots to gain a better understanding of animal locomotion, and in using inspiration from biology to design novel types of robots and controllers.

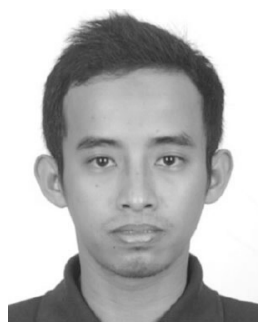

Azhar Aulia Saputra received Bachelor of Applied Science from Politeknik Elektronika Negeri Surabaya, Indonesia, in 2014, and the M.Eng. degree from Tokyo Metropolitan University, Tokyo, Japan, in 2018.

He is the JSPS Research Fellow DC1 from 2018 to 2021 . He has defended his doctoral thesis with Tokyo Metropolitan University in 2021. His research interests are intelligent control systems, locomotion systems, neuro-cognitive robotics, and legged robotics.

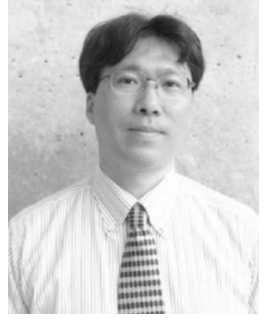

Naoyuki Kubota (Member, IEEE) received the M.Eng. degree from Hokkaido University, Hokkaido, Japan, in 1994, and the D.E. degree from Nagoya University, Nagoya, Japan, in 1997.

$\mathrm{He}$ joined the Osaka Institute of Technology, Osaka, Japan, in 1997. In 2000, he joined the Department of Human and Artificial Intelligence Systems, Fukui University, Fukui, Japan, as an Associate Professor. He joined the Department of Mechanical Engineering, Tokyo Metropolitan University, Tokyo, Japan, in 2004, where he is a Professor with the Department of System Design. 